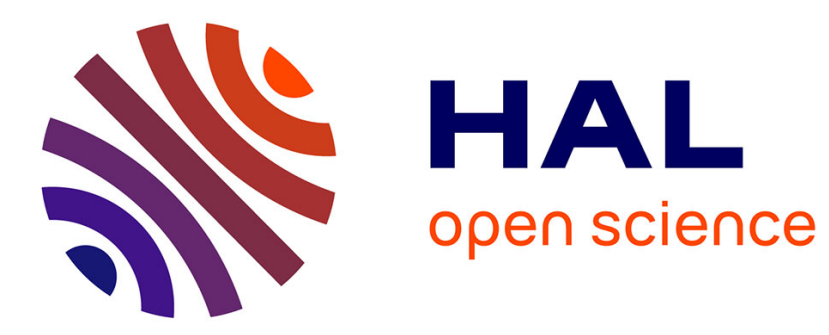

\title{
Simplified Random-Walk-Model-Based Kalman Filter for Slow to Moderate Fading Channel Estimation in OFDM Systems
}

Huaqiang Shu, Laurent Ros, Eric Pierre Simon

\section{- To cite this version:}

Huaqiang Shu, Laurent Ros, Eric Pierre Simon. Simplified Random-Walk-Model-Based Kalman Filter for Slow to Moderate Fading Channel Estimation in OFDM Systems. IEEE Transactions on Signal Processing, 2014, 62 (15), pp.4006-4017. 10.1109/TSP.2014.2332447 . hal-01021519

\author{
HAL Id: hal-01021519 \\ https://hal.science/hal-01021519
}

Submitted on 9 Jul 2014

HAL is a multi-disciplinary open access archive for the deposit and dissemination of scientific research documents, whether they are published or not. The documents may come from teaching and research institutions in France or abroad, or from public or private research centers.
L'archive ouverte pluridisciplinaire HAL, est destinée au dépôt et à la diffusion de documents scientifiques de niveau recherche, publiés ou non, émanant des établissements d'enseignement et de recherche français ou étrangers, des laboratoires publics ou privés. 


\title{
Simplified Random-Walk-Model-Based Kalman Filter for Slow to Moderate Fading Channel Estimation in OFDM Systems
}

\author{
Huaqiang Shu ${ }^{* \dagger}$, Laurent Ros *, and Eric Pierre Simon ${ }^{\dagger}$
}

\begin{abstract}
This study deals with multi-path channel estimation for orthogonal frequency division multiplexing systems under slow to moderate fading conditions. Advanced algorithms exploit the channel time-domain correlation by using Kalman Filters (KFs) based on an approximation of the time-varying channel. Recently, it was shown that under slow to moderate fading, near optimal channel multi-path complex amplitude estimation can be obtained by using the integrated Random Walk (RW) model as the channel approximation. To reduce the complexity of the high-dimensional RW-KF for joint estimation of the multi-path complex amplitudes, we propose using a lower dimensional RWKF that estimates the complex amplitude of each path separately. We demonstrate that this amounts to a simplification of the joint multi-path Kalman gain formulation through the Woodbury's identities. Hence, this new algorithm consists of a superposition of independent single-path single-carrier KFs, which were optimized in our previous studies. This observation allows us to adapt the optimization to the actual multi-path multi-carrier scenario, to provide analytic formulae for the mean-square error performance and the optimal tuning of the proposed estimator directly as a function of the physical parameters of the channel (Doppler frequency, Signal-to-Noise-Ratio, Power Delay Profile). These analytic formulae are given for the first-, second-, and thirdorder RW models used in the KF. The proposed per-path KF is shown to be as efficient as the exact KF (i.e., the joint multipath KF), and outperforms the autoregressive-model-based KFs proposed in the literature.
\end{abstract}

Index Terms-Orthogonal frequency division multiplexing, channel estimation, Rayleigh fading, Jakes' spectrum, randomwalk model, Kalman filter.

\section{INTRODUCTION}

Orthogonal Frequency Division Multiplexing (OFDM) is an effective technique for alleviating frequency-selective channel effects in wireless communication systems. In this technique, a wideband frequency-selective channel is converted to a number of parallel narrow-band flat fading subchannels that are free of inter-symbol interference and inter-carrier interference (for negligible channel time variations within one OFDM symbol period $T$ ). For coherent detection of the information symbols, reliable estimation of the channel in OFDM systems is crucial.

Most of the conventional methods work in a symbol-bysymbol scheme [1]-[3] using the correlation of the channel only in the frequency domain; i.e., the correlation between the subchannels. More advanced algorithms are based on the

*GIPSA-Lab, Image and Signal Department, BP 46, 38402 Saint Martin d'Hères, France.

${ }^{\dagger}$ IEMN lab, TELICE group, 59655 Villeneuve d'Ascq, France.
Kalman Filter (KF), to also exploit the time-domain correlation. This paper deals with channel multi-path ComplexAmplitude (CA) estimators based on KFs.

KFs require a linear recursive state-space representation of the channel. However, the exact Clarke model does not admit such a representation. An approximation often used in the literature consists of approaching the fading process as autoregressive [4]. Hence, a widely used channel approximation is based on a first-order Auto-Regressive model (AR1), as recommended by [5], combined with a Correlation-Matching (CM) criterion to fix the AR1 coefficient. The KF channel estimator that results from this choice, hereafter called $\mathrm{AR} 1_{C M}-\mathrm{KF}$, has been used in several studies concerning various systems, such as in multiple-input-multiple-output systems [4], and in OFDM systems [5]-[8]. The $\mathrm{AR} 1_{C M}-\mathrm{KF}$ appears to be convenient for the very high mobility case, which leads to quasi-optimal channel estimation performance compared to lower bounds, as seen, for example, in [7], [8] (in these studies, the $\mathrm{AR} 1_{C M}-\mathrm{KF}$ is actually used to track the basis extension model coefficients of the high-speed channel). However, here we consider moderate normalized Doppler frequency $\left(f_{d} T\right)$ values; i.e., $f_{d} T \leq 10^{-2}$. This corresponds to low mobility $(\leq 50 \mathrm{~km} / \mathrm{h})$ with the actual systems such as Worldwide Interoperability for Microwave Access (WiMAX) Mobiles. However, with the development of the cognitive radio, lower carrier frequencies are investigated for future systems. For instance, VHF/UHF television broadcast bands from $54 \mathrm{MHz}$ to $862 \mathrm{MHz}$ [9] and aeronautical bands from $960 \mathrm{MHz}$ to $1215 \mathrm{MHz}$ are planned to be deployed. For a given $f_{d} T$, as the speed is inversely proportional to the carrier frequency, $f_{d} T$ values around $10^{-2}$ can correspond to a relative high mobility with such systems (hundreds of $\mathrm{km} / \mathrm{h}$ ). This prompts the need for a comprehensive study of channel estimation for $f_{d} T \leq 10^{-2}$. For this scenario, whereby the channel variation within one symbol duration can be neglected ( [3][6], [10]-[12]), the AR1 $C M$-KF estimator usually exploited in the literature is far from being effective [13]. This poor performance was recently explained analytically by [14]. A better tuning of the AR1 coefficient can focus on minimizing the estimation variance in the output of the KF, as proposed by [13] (with the analytic mean-square error (MSE) performance for a given Doppler and signal-to-noise ratio (SNR) scenario in [14]); i.e., using a Minimum Asymptotic Variance (MAV) criterion without imposing the CM constraint. The resulting estimator is referred to as $\mathrm{AR} 1_{M A V}-\mathrm{KF}$ in the present study. Equivalent asymptotic performance can also be obtained by a 
first-order Random Walk (RW)-model-based KF (RW1-KF) ( [15], [16]).

On the other hand, it has been shown recently that the MSE performance of a KF can still be improved by switching from the AR1 model to an integrated RW model (also called the integrated Brownian model) for the approximation model. A second-order RW model and a third-order RW model have been respectively considered in [17] and [18]. They take into account that the exact path $\mathrm{CA}$ continues in a given direction during several symbols for low $f_{d} T$, and shows a strong trend behaviour. The Kalman estimators based on these second-order and third-order models are here called the RW2-KF and RW3KF estimators, respectively.

The RW-KF estimators of the previously cited studies were designed for single-path channel estimation in single-carrier systems. In the present study, we consider multi-path channel estimation in multi-carrier systems (i.e., OFDM systems). In this context, we are interested in devising simplified methods compared to the high-dimensional KFs that perform joint estimation of the path CAs. Some simplified methods have lately been proposed in [19]-[23]. Reference [19] converts the vector of pilot subcarriers into $L$ multi-path values where $L$ is the number of multi-paths and applies a KF to each path. The VSSO approach (Vector State - Scalar Observation) is proposed in [20]-[23], where single-path KFs are used to separately track each tap of the discrete-time-equivalent (DTE) channel. This has the advantage of reducing the complexity of the joint estimation given by the VSVO (Vector State - Vector Observation) approach, reported in [24] and [25, Equations (18)-(21)]. In [22], it is theoretically shown that the VSSO approach and the VSVO approach attain the same performance in the case of Wide-Sense Stationary Uncorrelated Scattering (WSSUS) DTE channel. Like aforementioned ideas, we propose in this paper a structure that uses a lower-dimensional KF for each channel path. However, unlike those, our study

- is developed in the framework of parametric (physical) channel model with the assumption of primary acquisition of the path delays as in [7], [11], [12], [26]-[28], instead of the DTE channel framework in [20]-[23],

- provides analytic results on the tuning and performance of the proposed estimator,

- is based on the RW model-based KF (instead of the AR1 model).

Our study also permits to shed new light on the behavior of per-path Kalman estimators (per-path RW-KF) and their link with joint multi-path Kalman estimators, this additionally corroborates previous results of [22].

The per-path Kalman estimator is achieved in two steps. First, we need to define an error signal for each path. To do this, we use the least-square (LS) estimator of the path CAs obtained only from the current OFDM symbol. This first step explores the frequency-domain correlation of the channel and the knowledge of the delays to convert the primary observation at pilot frequencies into a primary (instantaneous) estimate of the path CAs. Second, we apply a low-dimensional RW-KF for each path, to exploit the time-domain correlation of the channel. In this work, we show how to use our previous results [15], [17], [18] obtained in single-path single-carrier systems to fix the approximation model parameters of the proposed perpath RW-KF. We will show through simulations that the proposed estimator provides as good a performance as the highdimensional KF, with reduced complexity in case the number of multi-path components is small compared to the number of pilot subcarriers. This condition is generally true and necessary to the VSSO method [21], [22]. Another interesting aspect of this study, in addition to being a comprehensive study, is that the expression of the asymptotic variance performance of the proposed estimator is provided for the first to third orders of the RW model.

This paper is organized as follows: Section II introduces the OFDM system model, the wireless channel model, and the estimation objective. In Section III, we start from the joint multi-path KF equations based on the auto-regressive and RW models, and then we transform the KF equations to summarize an equivalent form that gives a path-wise presentation of the KF. Then, a per-path RW-KF is proposed to allow independent analysis of each single path, which allows the optimization results obtained in the single-carrier single-path context to be applied directly. Section IV validates first the per-path $\mathrm{RW} r-\mathrm{KF}$, by comparison with a numerically optimized joint multi-path RWr-KF, and then the theoretical analysis and the performance in terms of the asymptotic MSE.

Notations: $[\mathbf{x}]_{k}$ denotes the $k$ th entry of the vector $\mathbf{x}$, and $[\mathbf{X}]_{m, n}$ denotes the $[m, n]$ th entry of the matrix $\mathbf{X}$ (indices begin from 1). The notation $\operatorname{diag}\{\mathbf{x}\}$ is a diagonal matrix with $\mathbf{x}$ on its main diagonal and blkdiag $\{\mathbf{X}, \mathbf{Y}\}$ is a block diagonal matrix with the matrices $\mathbf{X}$ and $\mathbf{Y}$ on its main diagonal. $\mathbf{I}_{N}$ is an $N \times N$ identity matrix. The symbols of $\{\cdot\}^{T},\{\cdot\}^{H}$ and $\otimes$ stand for the transpose, Hermitian and Kronecker production operators respectively.

\section{OFDM System, Channel Model AND Estimation OBJECTIVE}

\section{A. OFDM system model}

Let us consider an OFDM system with $N$ subcarriers, and a cyclic prefix length $N_{g}$. The duration of an OFDM symbol is $T=N_{T} T_{s}$, where $T_{s}$ is the sampling time and $N_{T}=N+N_{g}$. Let $\mathbf{x}_{(k)}$ be the sequence of transmitted elementary symbols of the $k$ th OFDM symbol.The $n$th element $\left[\mathbf{x}_{(k)}\right]_{n}(n=1, \ldots, N)$ is phase-shift $(M$-PSK) or quadrature amplitude modulated ( $M$-QAM) symbol transmitted on the sub-carrier with indice $n-1-\frac{N}{2}$. The sequence of transmitted symbols is assumed to be zero-mean and stationary with normalized variance: $\mathrm{E}\left\{\left|\left[\mathbf{x}_{(k)}\right]_{n}\right|^{2}\right\}=1$. After transmission over a slowly time-varying multi-path channel and fast Fourier transform demodulation, the $k$ th received OFDM symbol $\mathbf{y}_{(k)}$ is given by:

$$
\mathbf{y}_{(k)}=\mathbf{H}_{(k)} \mathbf{x}_{(k)}+\mathbf{w}_{(k)},
$$

where $\mathbf{w}_{(k)}$ is an $N \times 1$ zero-mean complex circular Gaussian noise vector with covariance matrix $\sigma_{\mathrm{w}}^{2} \mathbf{I}_{N}$, and $\mathbf{H}_{(k)}$ is an 
$N \times N$ diagonal matrix ${ }^{1}$ with its diagonal elements given by:

$$
\left[\mathbf{H}_{(k)}\right]_{n, n}=\frac{1}{N} \sum_{l=1}^{L}\left[\alpha_{(k)}^{(l)} \cdot e^{-j 2 \pi\left(\frac{n-1}{N}-\frac{1}{2}\right) \tau^{(l)}}\right],
$$

where $L$ is the total number of propagation paths, $\alpha_{(k)}^{(l)}$ is the CA of the $l$-th path at $k$ th OFDM symbol with variance $\sigma_{\alpha(l)}^{2}$ (with $\sum_{l=1}^{L} \sigma_{\alpha(l)}^{2}=1$ ), and $\tau^{(l)} \times T_{s}$ is the $l$-th delay $\left(\tau^{(l)}\right.$ is not necessarily an integer, but $\left.\tau^{(l)}<N_{g}\right)$. The $L$ individual elements of $\left\{\alpha_{(k)}^{(l)}\right\}(l=1, \ldots, L)$ are uncorrelated w.r.t. one another. So, we adopt a parametric channel model as in [11], [12] with a WSSUS assumption of this physical channel. Note that there exists a corresponding DTE channel with taps uniformly spaced every $T_{s}$, and this DTE channel might be correlated (see the link between physical and DTE channels in Appendix A). Using Equation (2), the observation model of Equation (1) can be re-written [11] as:

$$
\mathbf{y}_{(k)}=\operatorname{diag}\left\{\mathbf{x}_{(k)}\right\} \mathbf{F} \boldsymbol{\alpha}_{(k)}+\mathbf{w}_{(k)},
$$

where $\boldsymbol{\alpha}_{(k)}=\left[\begin{array}{lll}\alpha_{(k)}^{(1)} & \ldots & \alpha_{(k)}^{(L)}\end{array}\right]^{T}$ and $\mathbf{F}$ is an $N \times L$ Fourier matrix depending on the delay distribution, with elements given by: $[\mathbf{F}]_{k, l}=e^{-j 2 \pi\left(\frac{k-1}{N}-\frac{1}{2}\right) \tau^{(l)}}$.

We assume the "Rayleigh-Jakes" model [29] for the multipath channel, with Doppler frequency $f_{d}$. This means that the $L$ CAs $\alpha_{(k)}^{(l)}$ are independent wide-sense stationary zero-mean complex circular Gaussian processes, with the correlation coefficients for the time-lag $q$ given by:

$$
\mathrm{R}_{\alpha^{(l)}}^{(q)}=\mathrm{E}\left[\alpha_{(k)}^{(l)} \alpha_{(k-q)}^{(l) H}\right]=\sigma_{\alpha^{(l)}}^{2} J_{0}\left(2 \pi f_{d} T q\right),
$$

where $J_{0}$ is the zero-th order Bessel function of the first kind. For each given path $l$, the Jakes' Doppler spectrum with $f_{d}$ of the path CA $\alpha_{(k)}^{(l)}$ is defined as:

$$
\Gamma_{\alpha^{(l)}}(f)=\left\{\begin{array}{ccc}
\frac{\sigma_{\alpha}^{2}(l)}{\pi f_{d} \sqrt{1-\left(\frac{f}{f_{d}}\right)^{2}}}, & \text { if } & |f|<f_{d} \\
0, & \text { if } & |f| \geq f_{d} .
\end{array}\right.
$$

\section{B. Pilot pattern}

We use $N_{p}$ pilot sub-carriers, they are evenly inserted into the $N$ sub-carriers at the positions $\mathcal{P}=\left\{n_{p} \mid n_{p}=\right.$ $\left.(p-1) L_{f}+1, p=1, \ldots, N_{p}\right\}$ with $L_{f}$ as the distance between two adjacent pilots. The received pilot sub-carriers can be written as:

$$
\mathbf{y}_{\mathbf{p}(k)}=\operatorname{diag}\left\{\mathbf{x}_{\mathbf{p}(k)}\right\} \mathbf{F}_{\mathbf{p}} \boldsymbol{\alpha}_{(k)}+\mathbf{w}_{\mathbf{p}(k)}
$$

where $\mathbf{x}_{\mathbf{p}}, \mathbf{y}_{\mathbf{p}}$ and $\mathbf{w}_{\mathbf{p}}$ are $N_{p} \times 1$ vectors, which correspond to the sent and received data symbol, and the channel noise on the pilot sub-carriers, respectively. The $N_{p} \times L$ matrix $\mathbf{F}_{\mathbf{p}}$ is the Fourier matrix of the pilot sub-carriers, with elements given by: $\left[\mathbf{F}_{\mathbf{p}}\right]_{n_{p}, l}=e^{-j 2 \pi\left(\frac{n_{p}-1}{N}-\frac{1}{2}\right) \tau^{(l)}}$, where $n_{p} \in \mathcal{P}$.

\footnotetext{
${ }^{1}$ We assume in the present study that during one OFDM symbol time, the $\mathrm{CA}$ in each path can be considered as a constant.
}

\section{Bayesian Cramer-Rao bound}

For any estimator $\hat{\boldsymbol{\alpha}}_{(k)}$ of $\boldsymbol{\alpha}_{(k)}$, we define the asymptotic MSE (valid for $k \rightarrow \infty$ ) by:

$$
\sigma_{\epsilon}^{2}=\frac{1}{L} \cdot E\left\{\left(\boldsymbol{\alpha}_{(k)}-\hat{\boldsymbol{\alpha}}_{(k)}\right)^{H} \cdot\left(\boldsymbol{\alpha}_{(k)}-\hat{\boldsymbol{\alpha}}_{(k)}\right)\right\},
$$

The estimation objective is to approach the asymptotic MSE $\sigma_{\epsilon}^{2}$ to the Bayesian Cramer-Rao Bound (BCRB). The on-line BCRB for the estimation of $\boldsymbol{\alpha}_{(k)}$ from the present and previous observations over a multi-path Rayleigh fading channel and OFDM modulation was derived by [30] for data-aided and non-data-aided contexts. For the pilot-based observation set $\left[\mathbf{y}_{\mathbf{p}(1)}, \cdots, \mathbf{y}_{\mathbf{p}(k)}\right]$ in the present case, any unbiased estimator $\hat{\boldsymbol{\alpha}}_{(k)}$ should satisfy:

$$
\sigma_{\epsilon}^{2} \geq \operatorname{BCRB}_{(k)},
$$

where the on-line BCRB is calculated by:

$$
\operatorname{BCRB}_{(k)}=\frac{1}{L} \cdot \sum_{i=(k-1) L+1}^{k L}[\mathbf{B C R B}(k)]_{i, i},
$$

with

$$
\mathbf{B C R B}_{(k)}=\left(\operatorname{blkdiag}\{\mathbf{J}, \mathbf{J}, \cdots, \mathbf{J}\}+\mathbf{R}_{\boldsymbol{\alpha}}^{-1}\right)^{-1},
$$

where $\mathbf{J}=\frac{1}{\sigma_{\mathrm{w}}^{2}} \mathbf{F}_{\mathbf{p}}^{H} \mathbf{F}_{\mathbf{p}}$ is an $L \times L$ matrix, and the covariance matrix $\mathbf{R}_{\boldsymbol{\alpha}}$ of size $k L \times k L$ is defined by the elements:

$$
\begin{aligned}
& {\left[\mathbf{R}_{\boldsymbol{\alpha}}\right]_{i(l, q), i\left(l^{\prime}, q^{\prime}\right)}=} \\
& \left\{\begin{array}{cll}
R_{\alpha(l)}^{\left(q-q^{\prime}\right)} & \text { if } & l^{\prime}=l \in[1, L] ; q, q^{\prime} \in[0, k-1] \\
0 & \text { if } & l^{\prime} \neq l,
\end{array}\right.
\end{aligned}
$$

with $i(l, q)=1+(l-1)+q L$. In the simulation section, we will plot $\mathrm{BCRB}=\lim _{k \rightarrow \infty} \mathrm{BCRB}_{(k)}$ as a reference.

\section{RANDOM-WALK-MODEL-BASED KALMAN FILTERS}

\section{A. Joint multi-path KFs}

To design and apply a KF, the channel can be first approximated using a Gauss-Markov process to construct a statespace model [31]. For a slow to moderate fading channel, the path $\mathrm{CA} \alpha_{(k)}^{(l)}$ shows a strong trend behaviour within several successive OFDM symbols. Based on this, we use the (integrated) RW model instead of AR1 to approach the $\mathrm{CA}$ variations. The $r$ th-order $\mathrm{RW}$ model $(\mathrm{RW} r)$ indicates that the $(r-1)$ th derivative of the variable that is approximated by the finite difference method is modeled as a RW process. Therefore, for the RW1 model, the approximate process of $\alpha_{(k)}^{(l)}$, which is denoted as $\tilde{\alpha}_{(k)}^{(l)}$, is updated at symbol time by adding zero mean circular complex Gaussian state noise. The variance of the state noise is adjustable, and this is used as the model parameter to be tuned. The RW2 model includes a linear drift $\delta_{(k)}^{(l)}$, that is updated at symbol time by adding the state noise. The variance of the state noise should also be well calibrated, such that the drift (which is regarded as the slope of the CA) varies slowly with time, by taking into account the time variation of the channel. In expecting a more accurate estimation, the RW3 model is obtained by considering the first and second approximate derivatives of CA at the previous time 
TABLE I: List of terms in the KF state-space model

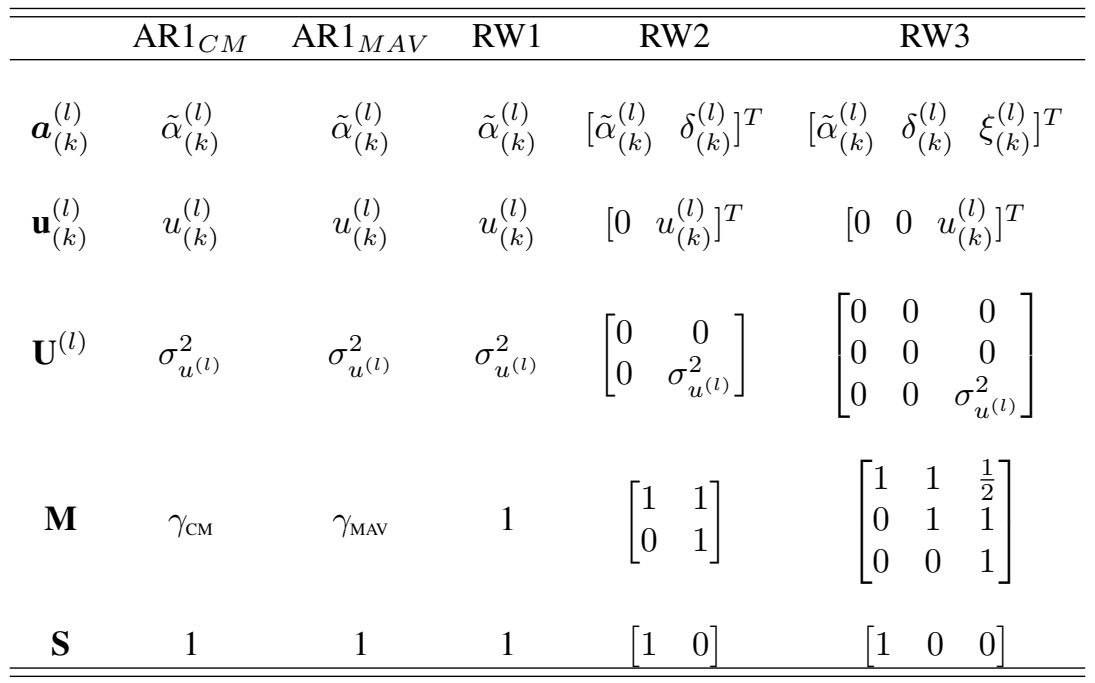

slot, and the second derivative is driven by the state noise. The third-order model has been widely applied to KF and phaselocked loops for phase tracking problems in satellite receivers [32].

The state model of the $L$-path CAs can be expressed in vector form as:

$$
\boldsymbol{a}_{(k)}=\mathcal{M} \boldsymbol{a}_{(k-1)}+\mathbf{u}_{(k)},
$$

where $\boldsymbol{a}_{(k)}=\left[\begin{array}{lll}\boldsymbol{a}_{(k)}^{(1) T} & \cdots & \boldsymbol{a}_{(k)}^{(L) T}\end{array}\right]^{T}$ with $\boldsymbol{a}_{(k)}^{(l)}$ as the state vector of the $l$-th path, $\mathbf{u}_{(k)}=\left[\begin{array}{lll}\mathbf{u}_{(k)}^{(1) T} & \cdots & \mathbf{u}_{(k)}^{(L) T}\end{array}\right]^{T}$ with $\mathbf{u}_{(k)}^{(l)}$ as the state noise vector of the $l$-th path, $\mathcal{M}=\mathbf{I}_{L} \otimes \mathbf{M}$ is the channel state evolution matrix. The path variables $\boldsymbol{a}_{(k)}^{(l)}$, $\boldsymbol{u}_{(k)}^{(l)}$ and the path state evolution matrix $\mathbf{M}$ are defined in Table I, according to the model order.

From Equation (6), we first derive the transmitted pilot symbol $\mathbf{x}_{\mathbf{p}(k)}$ from the received pilot symbol $\mathbf{y}_{\mathbf{p}(k)}$ as:

$$
\tilde{\mathbf{y}}_{\mathbf{p}(k)}=\mathbf{F}_{\mathbf{p}} \boldsymbol{\alpha}_{(k)}+\tilde{\mathbf{w}}_{\mathbf{p}(k)},
$$

where $\tilde{\mathbf{y}}_{\mathbf{p}(k)}$ and $\tilde{\mathbf{w}}_{\mathbf{p}(k)}$ are respectively defined by:

$$
\left[\tilde{\mathbf{y}}_{\mathbf{p}(k)}\right]_{p}=\frac{\left[\mathbf{y}_{(k)}\right]_{n_{p}}}{\left[\mathbf{x}_{(k)}\right]_{n_{p}}}, \quad\left[\tilde{\mathbf{w}}_{\mathbf{p}(k)}\right]_{p}=\frac{\left[\mathbf{w}_{(k)}\right]_{n_{p}}}{\left[\mathbf{x}_{(k)}\right]_{n_{p}}},
$$

with $p=1, \ldots, N_{p}$ and $n_{p}=(p-1) L_{f}+1$. Note that $\tilde{\mathbf{w}}_{\mathbf{p}}$ has the same covariance matrix as $\mathbf{w}_{\mathbf{p}}$, given that $\mathbf{x}_{\mathbf{p}(k)}$ is a quadrature phase shift keying symbol. We define the multipath selection matrix $\mathcal{S}=\mathbf{I}_{L} \otimes \mathbf{S}$ with $\mathbf{S}$ being given in Table I according to the model order. This matrix allows us to pass from the vector $\boldsymbol{a}_{(k)}$ to $\boldsymbol{\alpha}_{(k)}$ using $\boldsymbol{\alpha}_{(k)}=\mathcal{S} \boldsymbol{a}_{(k)}$. By defining $\mathbf{F}_{\mathbf{s}}=\mathbf{F}_{\mathbf{p}} \mathcal{S}$, we obtain from (12) the observation equation of the joint multi-path KF:

$$
\tilde{\mathbf{y}}_{\mathbf{p}(k)}=\mathbf{F}_{\mathbf{s}} \boldsymbol{a}_{(k)}+\tilde{\mathbf{w}}_{\mathbf{p}(k)} .
$$

According to Equation (13) and the state-space Equation (11), the joint multi-path KF is then given by:
Time update equations

$$
\begin{aligned}
& \hat{\boldsymbol{a}}_{(k \mid k-1)}=\mathcal{M} \hat{\boldsymbol{a}}_{(k-1 \mid k-1)}, \\
& \mathbf{P}_{(k \mid k-1)}=\mathcal{M} \mathbf{P}_{(k-1 \mid k-1)} \mathcal{M}^{T}+\mathbf{U},
\end{aligned}
$$

Measurement update equations

$$
\begin{aligned}
& \mathbf{K}_{(k)}=\mathbf{P}_{(k \mid k-1)} \mathbf{F}_{\mathbf{s}}^{H}\left(\mathbf{F}_{\mathbf{s}} \mathbf{P}_{(k \mid k-1)} \mathbf{F}_{\mathbf{s}}^{H}+\sigma_{\mathrm{w}}^{2} \mathbf{I}_{N_{p}}\right)^{-1}, \\
& \hat{\boldsymbol{a}}_{(k \mid k)}=\hat{\boldsymbol{a}}_{(k \mid k-1)}+\mathbf{K}_{(k)}\left(\tilde{\mathbf{y}}_{\mathbf{p}(k)}-\mathbf{F}_{\mathbf{s}} \hat{\boldsymbol{a}}_{(k \mid k-1)}\right), \\
& \mathbf{P}_{(k \mid k)}=\left(\mathbf{I}_{r L}-\mathbf{K}_{(k)} \mathbf{F}_{\mathbf{s}}\right) \mathbf{P}_{(k \mid k-1)},
\end{aligned}
$$

where $\hat{\boldsymbol{a}}_{(k \mid k-1)}$ and $\hat{\boldsymbol{a}}_{(k \mid k)}$ are the prediction and estimation vectors, respectively, of the path CAs, and they have the same vector structure as $\boldsymbol{a}_{(k)}$. The state noise variance matrix $\mathbf{U}$ is an $r L \times r L$ block-diagonal matrix, with $\mathbf{U}^{(l)},(l=1, \ldots, L)$ on its main diagonal, and the $r \times r$ matrix $\mathbf{U}^{(l)}$ is defined in Table I. The $r L \times r L$ matrices $\mathbf{P}_{(k \mid k-1)}$ and $\mathbf{P}_{(k \mid k)}$ are the prediction and estimation error variance matrices, respectively. The term of $\left(\tilde{\mathbf{y}}_{\mathbf{p}(k)}-\mathbf{F}_{\mathbf{s}} \hat{\boldsymbol{a}}_{(k \mid k-1)}\right)$ in Equation (17) is an $N_{p} \times 1$ error signal, which is updated by the received signal at the symbol rate. Each element of this signal represents the estimation error (from the prediction) of the corresponding sub-carrier.

In section III-B, we propose a KF that processes a path by path estimation (denoted per-path RW-KF). This allows us to directly apply the results of the single-path scenario. In the scope of establishing the link between the joint multipath KF and the per-path RW-KF, a new formulation of the joint multi-path KF equations should be provided. By using Woodbury's identities (the deduction of this transformation is given in Appendix B), Equation (16) can be rewritten as:

$$
\mathbf{K}_{(k)}=\mathbf{K}_{\mathbf{e q}(k)} \cdot\left(\mathbf{F}_{\mathbf{p}}^{H} \mathbf{F}_{\mathbf{p}}\right)^{-1} \mathbf{F}_{\mathbf{p}}^{H},
$$

with $\mathbf{K}_{\mathbf{e q}(k)}$ as an $r L \times L$ matrix defined by:

$$
\mathbf{K}_{\mathbf{e q}(k)}=\mathbf{P}_{(k \mid k-1)} \mathcal{S}^{H}\left(\mathcal{S} \mathbf{P}_{(k \mid k-1)} \mathcal{S}^{H}+\left(\mathbf{F}_{\mathbf{p}}^{H} \mathbf{F}_{\mathbf{p}}\right)^{-1} \sigma_{\mathrm{w}}^{2}\right)^{-1}
$$


Then, the measurement Equations (17) and (18) of the KF can be reformulated as:

$$
\begin{aligned}
& \hat{\boldsymbol{a}}_{(k \mid k)}=\hat{\boldsymbol{a}}_{(k \mid k-1)}+\mathbf{K}_{\mathbf{e q}(k)} \cdot \boldsymbol{v}_{\boldsymbol{\epsilon}(k)}, \\
& \mathbf{P}_{(k \mid k)}=\left(\mathbf{I}_{r L}-\mathbf{K}_{\mathbf{e q}(k)} \mathcal{S}\right) \mathbf{P}_{(k \mid k-1)},
\end{aligned}
$$

with the error signal defined by:

$$
\boldsymbol{v}_{\boldsymbol{\epsilon}(k)}=\left(\mathbf{F}_{\mathbf{p}}^{H} \mathbf{F}_{\mathbf{p}}\right)^{-1} \mathbf{F}_{\mathbf{p}}^{H} \cdot\left(\tilde{\mathbf{y}}_{\mathbf{p}(k)}-\mathbf{F}_{\mathbf{s}} \hat{\boldsymbol{a}}_{(k \mid k-1)}\right) .
$$

Then Equations (14), (15), (20), (21) and (22) form an equivalent $\mathrm{KF}$ with $\mathbf{K}_{\mathbf{e q}(k)}$ as the equivalent Kalman gain. Note that this equivalent $\mathrm{KF}$ gives a path-wise presentation based on the error signal $\boldsymbol{v}_{\boldsymbol{\epsilon}(k)}$ of size $L \times 1$, as defined in Equation (23), while the original error signal in Equation (17) is of size $N_{p} \times 1$. The $L$ elements of $\boldsymbol{v}_{\boldsymbol{\epsilon}(k)}$ correspond to the estimation errors on the $L$ corresponding paths. By observing this compressed version of the error signal (23), we find that it is actually the difference between the LS estimation of the CA calculated from the current received OFDM symbol $\hat{\boldsymbol{\alpha}}_{\mathrm{LS}(k)}$, and the last prediction of the CA $\hat{\boldsymbol{\alpha}}_{(k \mid k-1)}$ :

$$
\boldsymbol{v}_{\boldsymbol{\epsilon}(k)}=\hat{\boldsymbol{\alpha}}_{\mathbf{L s}(k)}-\hat{\boldsymbol{\alpha}}_{(k \mid k-1)},
$$

since the LS estimate is obtained by [28], [33, Sec. III.B.1] :

$$
\hat{\boldsymbol{\alpha}}_{\mathbf{L S}(k)}=\left(\mathbf{F}_{\mathbf{p}}^{H} \mathbf{F}_{\mathbf{p}}\right)^{-1} \mathbf{F}_{\mathbf{p}}^{H} \tilde{\mathbf{y}}_{\mathbf{p}(k)} .
$$

Hence, each component of the error signal vector $\boldsymbol{v}_{\boldsymbol{\epsilon}(k)}$ in Equation (24) (instead of Equation (23)) corresponds to one individual path. However, the joint multi-path KF in Equation (21) does not process each path independently, due to the presence of $\left(\mathbf{F}_{\mathbf{p}}^{H} \mathbf{F}_{\mathbf{p}}\right)^{-1}$ in $\mathbf{K}_{\mathbf{e q}(k)}$ (see Equation (20)). This matrix depends on the power delay profile of the channel.

\section{B. The per-path $K F$ and its interpretation}

In this section, we present our less complex per-path RW$\mathrm{KF}$ solution that is based on independent processing of the paths. Firstly, a KF for a single path is proposed to filter the LS estimate of the path CA. Secondly, based on this, the global per-path KF formulation for the $L$ paths is presented. Finally, the link with the joint multi-path KF is established.

1) Single-path KF: We use the LS estimation of $\boldsymbol{\alpha}$ (defined in Equation (25)) instead of $\tilde{\mathbf{y}}_{\mathbf{p}(k)}$ as the input signal to reformulate the $\mathrm{KF}$, and impose independent processing of the $L$ paths. The $l$-th element of $\hat{\boldsymbol{\alpha}}_{\mathrm{LS}(k)}$, denoted by $\hat{\alpha}_{\mathrm{LS}(k)}^{(l)}$, corresponds to the LS estimation of the $l$-th path CA. Also, let us define the LS estimation error as the loop noise applied on the per-path KF, denoted by $\mathbf{w}_{\mathrm{LS}}$. Then, the state-space model of the per-path KF for the $l$-th path is given by:

$$
\begin{aligned}
& \hat{\alpha}_{\mathrm{LS}(k)}^{(l)}=\alpha_{(k)}^{(l)}+\mathrm{w}_{\mathrm{LS}(k)}^{(l)}, \\
& \boldsymbol{a}_{(k)}^{(l)}=\mathbf{M} \boldsymbol{a}_{(k-1)}^{(l)}+\mathbf{u}_{(k)}^{(l)},
\end{aligned}
$$

where $\mathrm{w}_{\mathrm{LS}(k)}^{(l)}=\left[\left(\mathbf{F}_{\mathbf{p}}^{H} \mathbf{F}_{\mathbf{p}}\right)^{-1} \mathbf{F}_{\mathbf{p}}^{H} \tilde{\mathrm{w}}_{\mathbf{p}(k)}\right]_{l}$ in the per-path observation equation (26) is the $l$-th element of the loop noise $\mathbf{w}_{\mathbf{L S}}$ with variance:

$$
\sigma_{\mathbf{L S}}^{2}(l)=\sigma_{\mathrm{w}}^{2} \cdot\left[\left(\mathbf{F}_{\mathbf{p}}^{H} \mathbf{F}_{\mathbf{p}}\right)^{-1}\right]_{l, l} .
$$

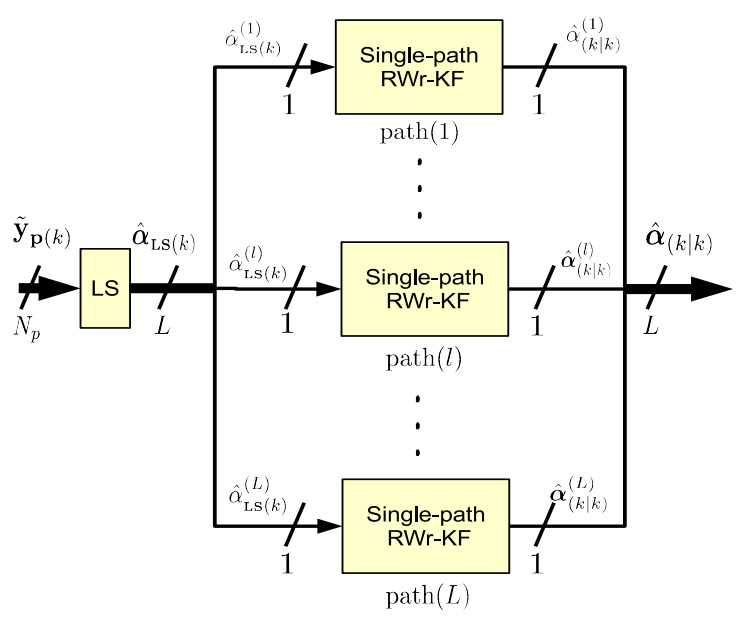

Fig. 1: Per-path KF structure

The single-path KF for the $l$-th path can thus be written as [18]:

Time update equations

$$
\begin{aligned}
& \hat{\boldsymbol{a}}_{(k \mid k-1)}^{(l)}=\mathbf{M} \hat{\boldsymbol{a}}_{(k-1 \mid k-1)}^{(l)}, \\
& \mathbf{P}_{(k \mid k-1)}^{(l)}=\mathbf{M} \mathbf{P}_{(k-1 \mid k-1)}^{(l)} \mathbf{M}^{T}+\mathbf{U}^{(l)},
\end{aligned}
$$

Measurement update equations

$$
\begin{aligned}
& \mathbf{K}_{(k)}^{(l)}=\frac{\mathbf{P}_{(k \mid k-1)}^{(l)} \mathbf{S}^{T}}{\mathbf{S P}_{(k \mid k-1)}^{(l)} \mathbf{S}^{T}+\sigma_{\mathrm{LS}}^{2}(l)}, \\
& \hat{\boldsymbol{a}}_{(k \mid k)}^{(l)}=\hat{\boldsymbol{a}}_{(k \mid k-1)}^{(l)}+\mathbf{K}_{(k)}^{(l)}\left(\hat{\alpha}_{\mathrm{LS}(k)}^{(l)}-\mathbf{S} \hat{\boldsymbol{a}}_{(k \mid k-1)}^{(l)}\right), \\
& \mathbf{P}_{(k \mid k)}^{(l)}=\left(\mathbf{I}_{r}-\mathbf{K}_{(k)}^{(l)} \mathbf{S}\right) \mathbf{P}_{(k \mid k-1)}^{(l)} .
\end{aligned}
$$

Note that in the denominator of (31), $\mathbf{S P}_{(k \mid k-1)}^{(l)} \mathbf{S}^{T}$ reduces to the first element of the matrix $\mathbf{P}_{(k \mid k-1)}^{(l)}$.

2) The per-path $K F$ : We can merge previous single-path $\mathrm{KF}$ equations for the $L$ paths of the channel into a global formulation, as illustrated in Figure 1. This is done for comparison with the joint multi-path KF. The prediction and estimation vectors of the multi-path channel state $\hat{\boldsymbol{a}}_{(k \mid k-1)}, \hat{\boldsymbol{a}}_{(k \mid k)}$, the state noise variance matrix $\mathbf{U}$, the state evolution matrix $\mathcal{M}$, and the selection matrix $\mathcal{S}$ remain the same structure as defined in the joint multi-path KF. As the processing of the paths is independent, we get the multi-path Kalman gain simply by overlaying the $r \times 1$ single-path Kalman gains as:

$$
\mathbf{K}_{\mathbf{p p}(k)}=\operatorname{blkdiag}\left\{\mathbf{K}_{(k)}^{(1)} \cdots \mathbf{K}_{(k)}^{(L)}\right\}
$$

and we obtain the per-path KF equations as:

Time update equations

$$
\begin{aligned}
& \hat{\boldsymbol{a}}_{(k \mid k-1)}=\mathcal{M} \hat{\boldsymbol{a}}_{(k-1 \mid k-1)}, \\
& \mathbf{P}_{(k \mid k-1)}=\mathcal{M} \mathbf{P}_{(k-1 \mid k-1)} \mathcal{M}^{T}+\mathbf{U},
\end{aligned}
$$


TABLE II: Closed-form expressions for the per-path RW $r$-KF in steady-state mode for the $l$-th path ( [15] [16] for $r=1$, and [17] [18] for $r=2,3$, respectively), under the assumption $f_{d} T \ll 1$ and $\sigma_{u^{(l)}} \ll \sigma_{\mathbf{L S}}{ }^{(l)}$

\begin{tabular}{|c|c|c|c|}
\hline & RW1 & RW2 & RW3 \\
\hline $\mathbf{K}_{(\infty)}^{(l)}$ & $\frac{\sigma_{u(l)}}{\sigma_{\mathbf{L S}}(l)}$ & 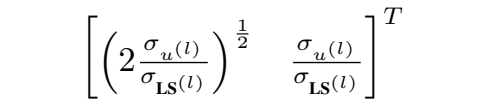 & {$\left[2\left(\frac{\sigma_{u}(l)}{\sigma_{\mathbf{L S}}(l)}\right)^{\frac{1}{3}} \quad 2\left(\frac{\sigma_{u}(l)}{\sigma_{\mathbf{L S}}(l)}\right)^{\frac{2}{3}} \quad \frac{\sigma_{u}(l)}{\sigma_{\mathbf{L S}}(l)}\right]$} \\
\hline$\sigma_{\epsilon \alpha^{(l)}}^{2}$ & $2 \cdot\left(\pi f_{d} T\right)^{2} \cdot \frac{\sigma_{\mathbf{L S}}^{2}(l)}{\sigma_{u^{(l)}}^{2}} \cdot \sigma_{\alpha^{(l)}}^{2}$ & $6 \cdot\left(\pi f_{d} T\right)^{4} \cdot \frac{\sigma_{\mathbf{L S}(l)}^{2}}{\sigma_{u}^{2}(l)} \cdot \sigma_{\alpha^{(l)}}^{2}$ & $20 \cdot\left(\pi f_{d} T\right)^{6} \cdot \frac{\sigma_{\mathbf{L S}(l)}^{2}}{\sigma_{u}^{2}(l)} \cdot \sigma_{\alpha^{(l)}}^{2}$ \\
\hline$\sigma_{\epsilon \mathrm{W}}^{2}(l)$ & $\frac{\sigma_{u(l)} \sigma_{\mathbf{L S}(l)}}{2}$ & $\frac{3}{4} \cdot\left(2 \sigma_{u^{(l)}}\right)^{\frac{1}{2}} \sigma_{\mathbf{L S}}^{\frac{3}{2}}$ & $\frac{5}{3} \sigma_{u^{(l)}}^{\frac{1}{3}} \sigma_{\mathbf{L S}^{(l)}}^{\frac{5}{3}}$ \\
\hline$\sigma_{u^{(l)} \text { opt }}^{2}$ & $4 \cdot\left[\left(\pi f_{d} T\right)^{4} \sigma_{\alpha^{(l)}}^{4} \sigma_{\mathbf{L S}}^{2}\right]^{\frac{1}{3}}$ & {$\left[2^{18}\left(\pi f_{d} T\right)^{16} \sigma_{\alpha^{(l)}}^{8} \sigma_{\mathbf{L S}}^{2}\right]^{\frac{1}{5}}$} & {$\left[3^{12} \cdot 2^{18}\left(\pi f_{d} T\right)^{36} \sigma_{\alpha^{(l)}}^{12} \sigma_{\mathbf{L S}^{(l)}}^{2}\right]^{\frac{1}{7}}$} \\
\hline$\sigma_{\epsilon^{(l)} \min }^{2}$ & $\frac{3}{2} \cdot\left(\pi f_{d} T \cdot \sigma_{\mathbf{L S}(l)}^{2}\right)^{\frac{2}{3}} \cdot\left(\sigma_{\alpha^{(l)}}^{2}\right)^{\frac{1}{3}}$ & $\frac{15}{8} \cdot\left(\sqrt{2} \pi f_{d} T \cdot \sigma_{\mathbf{L S}^{(l)}}^{2}\right)^{\frac{4}{5}} \cdot\left(\sigma_{\alpha^{(l)}}^{2}\right)^{\frac{1}{5}}$ & $\frac{35}{16} \cdot\left(\frac{16}{9} \pi f_{d} T \cdot \sigma_{\mathbf{L S}^{(l)}}^{2}\right)^{\frac{6}{7}} \cdot\left(\sigma_{\alpha^{(l)}}^{2}\right)^{\frac{1}{7}}$ \\
\hline
\end{tabular}

Measurement update equations

$$
\begin{aligned}
& \mathbf{K}_{\mathbf{p p}(k)}=\mathbf{P}_{(k \mid k-1)} \mathcal{S}^{H}\left(\mathcal{S} \mathbf{P}_{(k \mid k-1)} \mathcal{S}^{H}\right. \\
& \left.+\operatorname{diag}\left\{\left[\begin{array}{lll}
\sigma_{\mathbf{L S}}^{2} & \cdots & \sigma_{\mathbf{L S}}^{2(L)}
\end{array}\right]\right\}\right)^{-1} \\
& \hat{\boldsymbol{a}}_{(k \mid k)}=\hat{\boldsymbol{a}}_{(k \mid k-1)}+\mathbf{K}_{\mathbf{p p}(k)} \cdot \boldsymbol{v}_{\boldsymbol{\epsilon}(k)} \text {, } \\
& \mathbf{P}_{(k \mid k)}=\left(\mathbf{I}_{r L}-\mathbf{K}_{\mathbf{p p}(k)} \mathcal{S}\right) \mathbf{P}_{(k \mid k-1)} \text {, }
\end{aligned}
$$

with the time update equations (35) and (36) repeated from Equations (14) and (15), and with the error signal $\boldsymbol{v}_{\boldsymbol{\epsilon}(k)}$ defined in (23), or in (24). Note that in (37), $\mathbf{P}_{(k \mid k-1)} \mathcal{S}^{H}$ is an $r L \times L$ block-diagonal matrix, and $\mathcal{S} \mathbf{P}_{(k \mid k-1)} \mathcal{S}^{H}$ is an $L \times L$ diagonal matrix.

3) Comparison with the joint multi-path $K F$ : Due to the earlier defined block-diagonal matrix $\mathbf{K}_{\mathbf{p p}(k)}$ of Equation (34), when choosing a block-diagonal matrix for the initialization of $\mathbf{P}_{(-1 \mid-1)}$, it is easy to find that $\mathbf{P}_{(k \mid k-1)}$ and $\mathbf{P}_{(k \mid k)}$ are now block-diagonal matrices with $\mathbf{P}_{(k \mid k-1)}^{(l)}$ and $\mathbf{P}_{(k \mid k)}^{(l)}, \quad(l=$ $1, \ldots, L)$ on their main diagonals, respectively. By comparing the joint multi-path KF Equations (14), (15), (19) (22), and the per-path KF Equations $(35) \sim(39)$, we find that the only difference between these lies in the equations of Kalman gain: the joint multi-path KF exploits the covariance matrix of the loop noise $\left(\mathbf{F}_{\mathbf{p}}^{H} \mathbf{F}_{\mathbf{p}}\right)^{-1} \sigma_{\mathrm{w}}^{2}$ in (20), while the per-path KF considers only the main diagonal elements of $\left(\mathbf{F}_{\mathbf{p}}^{H} \mathbf{F}_{\mathbf{p}}\right)^{-1} \sigma_{\mathrm{w}}^{2}$ in (37). This is why our proposed per-path KF can be viewed as an approximation of the joint multi-path KF.

4) Comparison with the literature results: From the previous analysis we can conclude that the joint multi-path KF and the per-path $\mathrm{KF}$ are strictly equivalent if $\mathbf{F}_{\mathbf{p}}^{H} \mathbf{F}_{\mathbf{p}}$ is a diagonal matrix. However, $\mathbf{F}_{\mathbf{p}}^{H} \mathbf{F}_{\mathbf{p}}$ is not such a diagonal matrix in general, unless the physical multi-path delays $\tau^{(l)} T_{s}$ are multiples of the sample time $T_{s}$, yielding $\mathbf{F}_{\mathbf{p}}^{H} \mathbf{F}_{\mathbf{p}}=N_{p} \mathbf{I}_{L}$ (see Appendix A). Having delays multiple of $T_{s}$ also means that the DTE channel is uncorrelated (see the correspondence between the parametric channel model and the DTE channel model). Hence, our result corroborates that of the latest reference [22] stating the equivalence between (vectorial) VSVO KF and (scalar) VSSO KF under the condition that the DTE channel is uncorrelated (WSSUS).

The per-path KF applied in this condition has been analyzed in [21]-[23] (called VSSO KF associate to a DTE channel channel). But in practice, the physical multi-path delays are not ensured to be multiples of $T_{s}$, thus $\mathbf{F}_{\mathbf{p}}^{H} \mathbf{F}_{\mathbf{p}} \neq N_{p} \mathbf{I}_{L}$, or equivalently the DTE channel is correlated. The following $\mathrm{KF}$ analysis will consider uncorrelated and correlated DTE channels, and will bring new analytical results for both channel conditions.

\section{Asymptotic mean-square error of the per-path $K F$}

We aim to find the asymptotic MSE, $\sigma_{\epsilon}^{2}$, as defined in Equation (7), for the proposed unbiased estimator.

In the single-path scenarios, the asymptotic MSE expressions of the RWr-KF $(r=1,2,3)$ were deduced in our previous studies [15]-[18]. These results can be applied to the per-path $\mathrm{KF}$ as it deals with each path independently, in the same way as a single-path channel scenario according to Equation (26). However, in the present study, the loop noise variance $\sigma_{\mathbf{L S}(l)}^{2}$ represents the observation noise variance in our previous studies.

The variance of estimation error is then comprised of two parts, one of which comes from the variation of the parameter $\boldsymbol{\alpha}$, and the other comes from the input loop noise $\mathbf{w}_{\mathbf{L S}}$. Thus for a given path $l$, we have:

$$
\sigma_{\epsilon^{(l)}}^{2}=\sigma_{\epsilon \alpha^{(l)}}^{2}+\sigma_{\epsilon \mathrm{W}^{(l)}}^{2} .
$$

The component $\sigma_{\epsilon \alpha^{(l)}}^{2}$ (i.e., the dynamic error variance) results from the high-pass filtering of the input CA $\alpha_{(k)}^{(l)}$, which can be expressed in the frequency-domain, by:

$$
\sigma_{\epsilon \alpha^{(l)}}^{2}=\int_{-\frac{1}{2 T}}^{+\frac{1}{2 T}} \Gamma_{\alpha^{(l)}}(f) \cdot\left|1-\mathcal{L}\left(e^{j 2 \pi f T}\right)\right|^{2} d f,
$$

where $\mathcal{L}\left(e^{j 2 \pi f T}\right)$ is the low-pass transfer function (expressed in the $z$-domain, with here $z=e^{j 2 \pi f T}$ ) of the steady-state $\mathrm{RW} r$-KF. The expressions of $\mathcal{L}(z)$ can be found in [15] [16] 
for $r=1$, and in [17] [18] for $r=2,3$. It should be noted that $\mathcal{L}(z)$ depends on the elements of the Kalman gain vector $\mathbf{K}_{(k)}^{(l)}$ of size $r \times 1$ obtained for the steady-state mode $(k \rightarrow \infty)$. The previously cited studies give the approximate closed-form expressions of $\mathbf{K}_{(\infty)}^{(l)}$ with respect to the ratio of the state noise to the loop noise standard deviations, $\frac{\sigma_{u}(l)}{\sigma_{\mathbf{L S}}(l)}$, as reported in Table II. Note that all these formulae are obtained under the following two assumptions: a low normalized Doppler frequency $f_{d} T \ll 1$ and a weak state noise standard deviation of the KF compared to the observation noise $\left(\sigma_{u^{(l)}} \ll \sigma_{\mathbf{L S}}(l)\right)$. This latter assumption means that we have a low Kalman gain (first element) $\left[\mathbf{K}_{(\infty)}^{(l)}\right]_{1} \ll 1$. The component $\sigma_{\epsilon \mathrm{W}}^{2}$ (i.e., the static error variance) results from the low-pass filtering of the input loop noise, and is expressed by:

$$
\sigma_{\epsilon \mathbf{W}^{(l)}}^{2}=\sigma_{\mathbf{L S}(l)}^{2} \cdot T \int_{-\frac{1}{2 T}}^{+\frac{1}{2 T}}\left|\mathcal{L}\left(e^{j 2 \pi f T}\right)\right|^{2} d f .
$$

The static error variance is also a function of the state noise variance $\sigma_{u^{(l)}}$, as reported in Table II.

Then, $\sigma_{u^{(l)}}$ is the parameter which permits us to tune the per-path RW-KF. To do this, we find the optimum value $\sigma_{u^{(l)} \text { opt }}^{2}$ for which the per-path MSE $\sigma_{\epsilon^{(l)}}^{2}$ is minimum. This minimum value is denoted $\sigma_{\epsilon^{(l)} \text { min }}^{2}$. The dynamic error variance $\sigma_{\epsilon \alpha^{(l)}}^{2}$, the static error variance $\sigma_{\epsilon \mathrm{W}^{(l)}}^{2}$, the optimized path state noise for the RWr model $\sigma_{u^{(l)} \text { opt }}^{2}$, and the corresponding minimized asymptotic MSE $\sigma_{\epsilon^{(l)} \text { min }}^{2}$ are summarized in Table II. It should be noted that these amounts depend on the multipath channel delay profile, via the path loop noise variance $\sigma_{\mathrm{LS}^{(l)}}^{2}$ (see Equation (28)). The global mean MSE (per path) of the channel estimation is then calculated by:

$$
\sigma_{\epsilon \min }^{2}=\frac{1}{L} \sum_{l=1}^{L} \sigma_{\epsilon^{(l)} \min }^{2} .
$$

For interpretation, we split the latest formula into several contributions as follows:

$$
\sigma_{\epsilon \text { min }}^{2}=C_{r} \cdot\left(f_{d} T\right)^{\frac{2 r}{2 r+1}} \cdot\left(\sigma_{\mathrm{w}}^{2}\right)^{\frac{2 r}{2 r+1}} \cdot \beta_{r}
$$

where $C_{r}$ is a constant related to the KF order. For $r=1,2,3$, we define respectively: $C_{1}=\frac{3}{2} \pi^{\frac{2}{3}}, C_{2}=\frac{15}{8}(\sqrt{2} \pi)^{\frac{4}{5}}, C_{3}=$ $\frac{35}{16}\left(\frac{16}{9} \pi\right)^{\frac{6}{7}}$. The coefficient $\beta_{r}(r=1,2,3)$ is a noise factor that depends on the channel PDP defined as follows:

$$
\beta_{r}=\frac{1}{L} \sum_{l=1}^{L}\left\{\left[\left(\mathbf{F}_{\mathbf{p}}^{H} \mathbf{F}_{\mathbf{p}}\right)^{-1}\right]_{l, l}^{\frac{2 r}{2 r+1}} \cdot\left(\sigma_{\alpha^{(l)}}^{2}\right)^{\frac{1}{2 r+1}}\right\} .
$$

This new formulation (44) clearly shows a slope of $\frac{2 r}{2 r+1}$ in logarithmic scale w.r.t. the channel noise variance $\sigma_{\mathrm{w}}^{2}$ and the normalized Doppler frequency $f_{d} T$ which will be confirmed in the simulation Section.

An interesting result ensuing from (45) is that for uncorrelated DTE channels, the performance no longer depends on the delay distribution (i.e., the set of integer values $\tau^{(l)}$ ), but only depends on the energy distribution of the paths. Indeed, when $\mathbf{F}_{\mathbf{p}}^{H} \mathbf{F}_{\mathbf{p}}=N_{p} \mathbf{I}_{L}$, the parameter $\beta_{r}$ becomes:

$$
\beta_{r}=\frac{1}{L \cdot N_{p}^{\frac{2 r}{2 r+1}}} \sum_{l=1}^{L}\left\{\left(\sigma_{\alpha^{(l)}}^{2}\right)^{\frac{1}{2 r+1}}\right\} .
$$

\section{Complexity}

As well as the availability of the analytical optimization, another key advantage of the per-path $\mathrm{RW} r-\mathrm{KF}$ is its simplicity compared to algorithms based on the joint multi-path KF. We illustrate the complexity of both of these algorithms by counting the complex multiplications in each iteration body (Equations (14) (18) for the joint multi-path $\mathrm{RW} r-\mathrm{KF}$, and Equations (35) (39) for the per-path $\mathrm{RW} r-\mathrm{KF}$ ) in Tables III and IV.

TABLE III: Number of complex multiplications of the joint

\begin{tabular}{|c|c|c|}
\hline Operation & Equation & Multiplications \\
\hline $\boldsymbol{\mathcal { M }} \hat{\boldsymbol{a}}_{(k-1 \mid k-1)}$ & (35) & $0\left(\frac{L}{4}\right.$ for $\left.\mathrm{RW} 3\right)$ \\
\hline $\mathcal{M} \mathbf{P}_{(k-1 \mid k-1)} \mathcal{M}^{T}$ & (36) & $0\left(r L^{2}\right.$ for $\left.\mathrm{RW} 3\right)$ \\
\hline $\mathbf{A}_{5}=\mathbf{P}_{(k \mid k-1)} \mathcal{S}^{H}$ & (37) & 0 \\
\hline $\mathbf{A}_{6} \stackrel{\left(N \mathbf{A}_{5}\right.}{=}$ & (37) & 0 \\
\hline 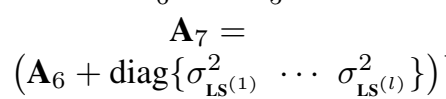 & $-1(37)$ & $L^{*}$ \\
\hline $\mathbf{A}_{5} \mathbf{A}_{7}$ & (37) & $r L$ \\
\hline $\mathbf{A}_{8}=\hat{\boldsymbol{\alpha}}_{\mathbf{L S}(k)}-\mathcal{S} \hat{\boldsymbol{a}}_{(k \mid k-1)}$ & (38) & $(L+1) N_{p}$ \\
\hline $\mathbf{K}_{\mathbf{p p}(k)} \mathbf{A}_{8}$ & (38) & $r L$ \\
\hline $\mathbf{K}_{\mathbf{p p}(k)} \mathbf{A}_{5}^{H}$ & (39) & $r^{2} L$ \\
\hline
\end{tabular}
multi-path RWr-KF within each iteration

\begin{tabular}{ccc}
\hline \hline Operation & Equation & Multiplications \\
\hline $\mathcal{M} \hat{\boldsymbol{a}}_{(k-1 \mid k-1)}$ & $(14)$ & $0\left(\frac{L}{4}\right.$ for RW3 $)$ \\
$\mathcal{M} \mathbf{P}_{(k-1 \mid k-1)} \mathcal{M}^{T}$ & $(15)$ & $0\left(r L^{2}\right.$ for RW3 $)$ \\
$\mathbf{A}_{1}=\mathbf{P}_{(k \mid k-1)} \mathbf{F}_{\mathbf{s}}^{H}$ & $(16)$ & $r N_{p} L^{2}$ \\
$\mathbf{A}_{2}=\mathbf{F}_{\mathbf{s}} \mathbf{A}_{1}$ & $(16)$ & $N_{p}^{2} L$ \\
$\mathbf{A}_{3}=\left(\mathbf{A}_{2}+\sigma_{\mathrm{w}}^{2} \mathbf{I}_{N_{p}}\right)$ & $(16)$ & $N_{p}^{3}$ \\
$\mathbf{A}_{1} \mathbf{A}_{3}$ & $(16)$ & $r L N_{p}^{2}$ \\
$\mathbf{A}_{4}=\tilde{\mathbf{y}}_{\mathbf{p}(k)}-\mathbf{F}_{\mathbf{s}} \hat{\boldsymbol{a}}_{(k \mid k-1)}$ & $(17)$ & $(L+1) N_{p}$ \\
$\mathbf{K}_{(k)} \mathbf{A}_{4}$ & $(17)$ & $r L N_{p}$ \\
$\mathbf{K}_{(k)} \mathbf{A}_{1}^{H}$ & $(18)$ & $r^{2} L^{2} N_{p}$ \\
\hline \hline
\end{tabular}

TABLE IV: Number of complex multiplications of the perpath RW $r$-KF within each iteration

* as the matrix is diagonal, the inversion operation is equivalent to division operations of the main diagonal elements, and here the division operation is considered to have the same order of complexity with multiplication.

Note that $\mathcal{M}, \mathcal{S}$ and $\mathbf{P}_{(k \mid k)}$ are real matrices, hence the first two equations of both of the RW-KFs are real operations, and thus the multiplications in these equations contribute to only a quarter of the complexity of the complex multiplications. Moreover, any multiplication with a matrix where the elements are only 0 and 1 does not contribute to the complexity; e.g., $\mathcal{M}$ for the first two orders and the matrix $\mathcal{S}$. Note that if the matrix $\mathbf{P}_{(k \mid k)}$ is initialized by a zero matrix, $\mathbf{P}_{(k \mid k)}$ and $\mathbf{P}_{(k \mid k-1)}$ for the per-path RW $r$-KF should be always kept block-diagonal (or diagonal for $r=1$ ). Hence in Table IV, 
TABLE V: Complexity comparison $(L=6)$

\begin{tabular}{ccccc}
\hline \hline \multirow{5}{*}{$N_{p}$} & $r$ & joint RWr-KF & per-path RW $r$-KF & Complexity saving \\
\hline \multirow{2}{*}{8} & 1 & 1790 & 72 & $95.98 \%$ \\
& 2 & 3074 & 102 & $96.68 \%$ \\
& 3 & 4790 & 122 & $96.99 \%$ \\
\hline \multirow{2}{*}{12} & 1 & 3966 & 96 & $97.58 \%$ \\
& 2 & 5730 & 126 & $97.8 \%$ \\
\hline \multirow{2}{*}{16} & 1 & 7926 & 168 & $97.88 \%$ \\
& 2 & 10114 & 120 & $98.44 \%$ \\
& 3 & 12982 & 150 & $98.52 \%$ \\
60 & 2 & 282210 & 192 & $98.52 \%$ \\
& 3 & 305124 & 384 & $99.85 \%$ \\
\hline \multirow{2}{*}{120} & 1 & 1901310 & 414 & $99.85 \%$ \\
& 2 & 1988610 & 456 & $99.85 \%$ \\
\hline \hline
\end{tabular}

$\mathbf{A}_{5}$ is block-diagonal, $\mathbf{A}_{6}$ and $\mathbf{A}_{7}$ are diagonal, so $\mathbf{A}_{5} \mathbf{A}_{7}$ needs $r L$ complex multiplications. The calculation of $\tilde{\mathbf{y}}_{\mathbf{p}(k)}$ has $N_{p}$ complex multiplications and $\hat{\boldsymbol{\alpha}}_{\mathbf{L s}(k)}$ has $N_{p} L$, since the calculation of $\left(\mathbf{F}_{\mathbf{p}}^{H} \mathbf{F}_{\mathbf{p}}\right)^{-1} \mathbf{F}_{\mathbf{p}}^{H}$ in $\hat{\boldsymbol{\alpha}}_{\mathrm{LS}(k)}$ can be done in advance and thus does not contribute to the complexity.

By summing Tables III and IV (here the complexity of the first two equations in Tables III and IV are not taken into account as they are negligible compared to the complex multiplications), we find that the joint multi-path $\mathrm{RW} r-\mathrm{KF}$ has $N_{p}^{3}+(r+1) L N_{p}^{2}+\left(r^{2} L^{2}+r L^{2}+r L+L+1\right) N_{p}$ complex multiplications, while the per-path $\mathrm{RW} r$-KF has only $\left[(r+1)^{2}+N_{p}\right] L$. Therefore, the joint multi-path $\mathrm{RW} r$-KF has a complexity of $O\left(N_{p}^{3}\right)$, while the per-path $\mathrm{RW} r$-KF has a complexity of $O\left(N_{p} L\right)$. The complexity depends only on the number of paths of the channel model, the number of pilot subcarriers and the estimator order $r$. The advantage of the perpath RW $r$-KF becomes evident in the case where the number of pilots $N_{p}>L$, which is most often the case in practice [34]. Furthermore, this condition is necessary for the VSSO method.

Table $\mathrm{V}$ gives a complexity comparison with $L=6$ (as in ETSI channel model used for 3GPP-UMTS standard) and with different $N_{p}$ (corresponding to different WiMAX profiles [35]). We find that for these scenarios, the complexity of perpath $\mathrm{RW} r-\mathrm{KF}$ is reduced by more than $95 \%$.

\section{Simulations}

In this simulation section, we use by default a 4QAMOFDM system with $N=128$ sub-carriers to validate the proposed approximate method and the analytic results. By default, the OFDM system has $N_{g}=16$ samples of CP, $N_{p}=16$ pilot sub-carriers in each transmitted OFDM symbol, and the system bandwidth is $1 / T_{s}=2 \mathrm{MHz}$. The GSM 6path outdoor channel model [36] was chosen as the default simulation channel, and its power delay profile is given in Table VI.

Figure 2 gives a comparison between the MSE performance of the joint multi-path RW-KFs and the per-path RW-KFs. In
TABLE VI: Power delay profile of the simulation channel

\begin{tabular}{|c|c|c|c|c|c|c|}
\hline Path & 1 & 2 & 3 & 4 & 5 & 6 \\
\hline \multicolumn{7}{|c|}{ correlated DTE channel (GSM): } \\
\hline$\tau^{(l)}$ & 0 & 0.4 & 1 & 3.2 & 4.6 & 10 \\
\hline$\sigma_{\alpha}^{2}(l)(\mathrm{dB})$ & -7.219 & -4.219 & -6.219 & -10.219 & -12.219 & -14.219 \\
\hline \multicolumn{7}{|c|}{ uncorrelated DTE channel: } \\
\hline$\tau^{(l)}$ & 0 & 1 & 2 & 3 & 4 & 10 \\
\hline$\sigma_{\alpha}^{2}(l)(\mathrm{dB})$ & -7.219 & -4.219 & -6.219 & -10.219 & -12.219 & -14.219 \\
\hline
\end{tabular}

this simulation, the per-path KFs are analytically optimized with state noise variance $\sigma_{u^{(l)} \text { opt }}^{2}$ given in Table II, while all the joint multi-path KFs are numerically optimized using a grid search around $\sigma_{u^{(l)} \text { opt }}^{2}$. We can observe that the joint multi-path KFs slightly outperform the per-path KFs, but the difference between both remains negligible. This validates the efficiency of the per-path KF.

Figure 3 shows the simulated asymptotic MSE (mean value per path) of the $\mathrm{AR} 1_{C M}-\mathrm{KF}$ and the RW-KF, the theoretical asymptotic MSE of the RW-KF, and the BCRB for $f_{d} T=$ $10^{-3}$ as a function of the SNR. We can observe that all the theoretical curves are very close to the simulated ones, except at high SNR where there is a sligth difference. This is explained by the fact that the theoretical formulae are obtained under the assumptions of a low Doppler frequency and low SNR scenarios (see Section III-C).

Figure 4 presents the MSE evolution as a function of $f_{d} T$. We can see clearly that the RW-KFs outperform the $\mathrm{AR} 1_{C M}$-KF. Moreover, the MSEs obtained by the simulation approximately coincide with the theoretical values (note that we have made approximations for the analytical expressions of the asymptotic MSE with the low $f_{d} T$ assumption; these approximations become poorer when $f_{d} T$ increases, and we observe an overestimated MSE for high $f_{d} T$, as shown in Fig. 4). For RW1-KF, RW2-KF and RW3-KF, the asymptotic MSE are respectively proportional to the $2 / 3,4 / 5,6 / 7$ powers of the $f_{d} T$, and are inversely proportional to the $2 / 3,4 / 5,6 / 7$ powers of the SNR, as argued in the theoretical analysis.

Figure 5 gives a comparison between a correlated and an uncorrelated DTE channel scenario. The PDP of these two channels are given in Table VI. The considered channel estimator is the per-path RW3-KF. As explained in Section III-B4, the physical delays for the uncorrelated DTE channel are multiples of $T_{s}$. The MSE obtained with the correlated DTE channel is greater than that obtained with the uncorrelated DTE channel. This is confirmed by the calculation of the loop noise factor $\beta_{r}$ (45) (see Section III-C) which gives $\beta_{3}=0.0719$ for the uncorrelated DTE channel and $\beta_{3}=0.173$ for the correlated DTE channel.

We simulate the Bit Error Rate (BER) performance for the proposed RW-KFs implemented with a zero-forcing equalizer in Figure 6. Here, we use 16-QAM modulation with and without coding and $N_{p}=8$ pilots for $f_{d} T=10^{-3}$. As other references, we have added the BER obtained for $\mathrm{AR} 1_{C M^{-}}$ $\mathrm{KF}$ estimator and that with perfect Channel State Information (CSI). Without coding, we can observe that the $\mathrm{AR} 1_{C M}$-KF curve and the RW1-KF curve are very close. Furthermore, 
the RW2-KF curve and the RW3-KF curve nearly coincide. At a BER target of $10^{-3}$, there is an SNR loss of about 2 $\mathrm{dB}$ between the RW2-KF and the RW1-KF. Then, we use a Non-Recursive Non-Systematic Convolutional (NRNSC) code $[5,7]_{8}$. This time, the RW1-KF curve is far from the AR $1_{C M^{-}}$ $\mathrm{KF}$ and closer to the RW2-KF curve. At a BER target of $10^{-5}$, there is an SNR loss of about $2.5 \mathrm{~dB}$ between the RW1-KF and the $\mathrm{AR} 1_{C M}$-KF curves, and only $0.5 \mathrm{~dB}$ between the RW1-KF and RW2-KF curves. To sum up, the BER of the $\mathrm{AR} 1_{C M}$ method is close to that of the RW method in the uncoded scenario while the difference is appreciable in the coded scenario. Indeed, a bad channel estimation (see MSE performance in Figure 3) has more impact on the detection in a coded scenario. It is interesting to note this, as coding could be always found in modern systems.

Now, we investigate an iterative detection and decoding scenario, in order to exploit soft data symbols in addition to pilot symbols. To do this, we use at the transmitter the classical bit-interleaved coded modulation scheme based on a convolutional code (the NRNSC code $[5,7]_{8}$ ) and a pseudorandom interleaver. At the receiver, we perform iterative soft channel estimation and channel decoding. The channel estimator/equalizer and the decoder exchange soft information in the form of Log-Likelihood Ratio (LLR) between them through a few iterations until convergence [37]. The channel decoder is based on the Soft-Output Viterbi Algorithm (SOVA). At the first iteration, only the pilots are used for channel estimation. Then, the next iterations exploit in addition the soft data symbols. The resulting MSE and BER are plotted in Fig. 7 as a function of $f_{d} T$ for $N_{p}=8$, QPSK symbols and an SNR of $10 \mathrm{~dB}$. The channel estimator is the RW1-KF. We notice a great performance improvement between the first iteration and the second iteration. Then, the algorithm converges. As expected, the MSE increases with an increasing $f_{d} T$, which is not necessarily the case for the BER. This is understood since the diversity is greater when $f_{d} T$ increases (see [38, Fig. 11]).

\section{CONCLUSIONS}

We have here proposed a low-dimensional KF solution for the estimation of the channel multi-path complex amplitudes. The considered approximation models for the KF are the RW models of the first, second and third orders. Our solution is a two-step solution: first, an error signal for each channel path is calculated with the LS criterion. Secondly, based on this error signal, a KF is applied to each path independently. This per-path KF solution explores the time-domain correlation of the channel, while the LS step exploits the frequency-domain correlation of the channel. We have shown how to apply the previous results we obtained for a single-path single-carrier to the multi-path multi-carrier context. This has allowed us to provide Tables with the optimal RW parameters, together with the theoretical formulae of the variance of the estimation error. Furthermore, we have demonstrated that our per-path KF solution can be interpreted as a simplified version of the more complex joint multi-path KF. This has been done through the Woodbury's identities. The simulation results show that the

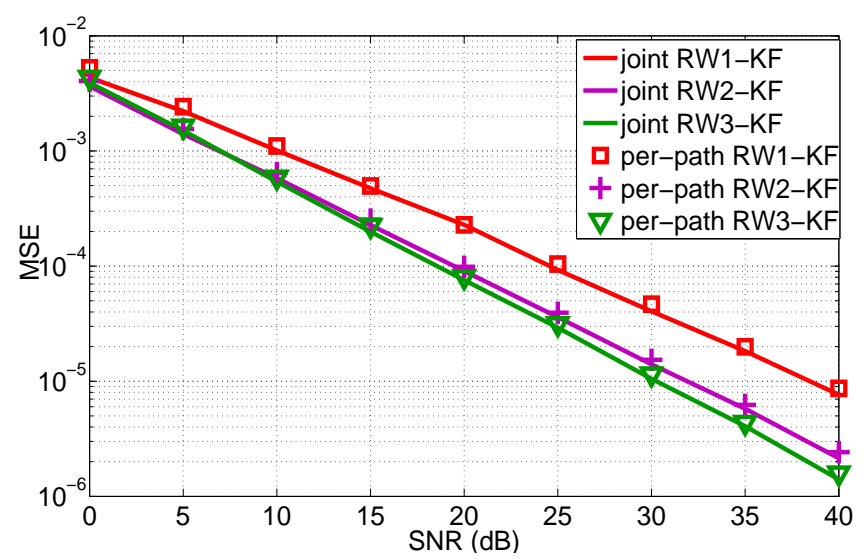

Fig. 2: Simulated MSE of the joint multi-path RW $r$-KF and the per-path $\mathrm{RW} r$-KF versus the SNR with $f_{d} T=10^{-3}, N_{p}=16$, GSM channel

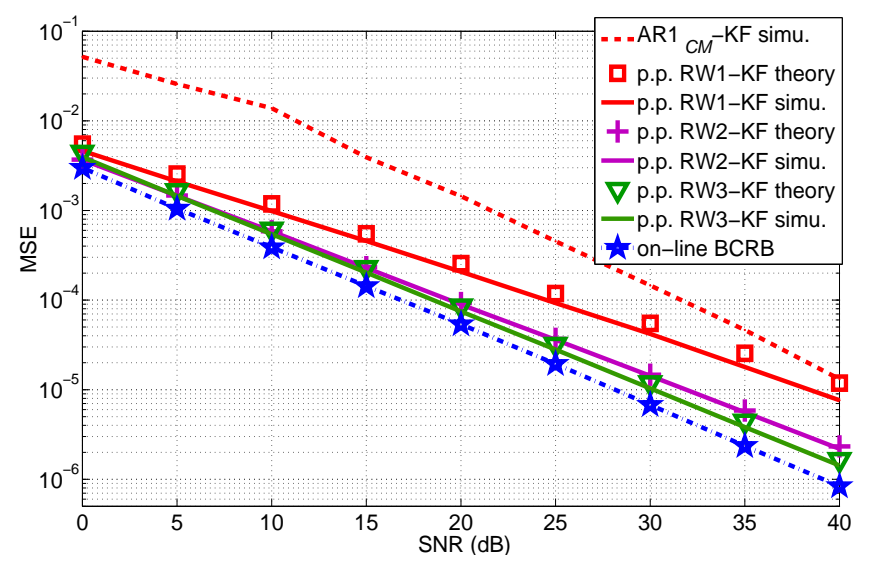

Fig. 3: MSE of the per-path KF versus the SNR with $f_{d} T=10^{-3}, N_{p}=$ 16 , GSM channel

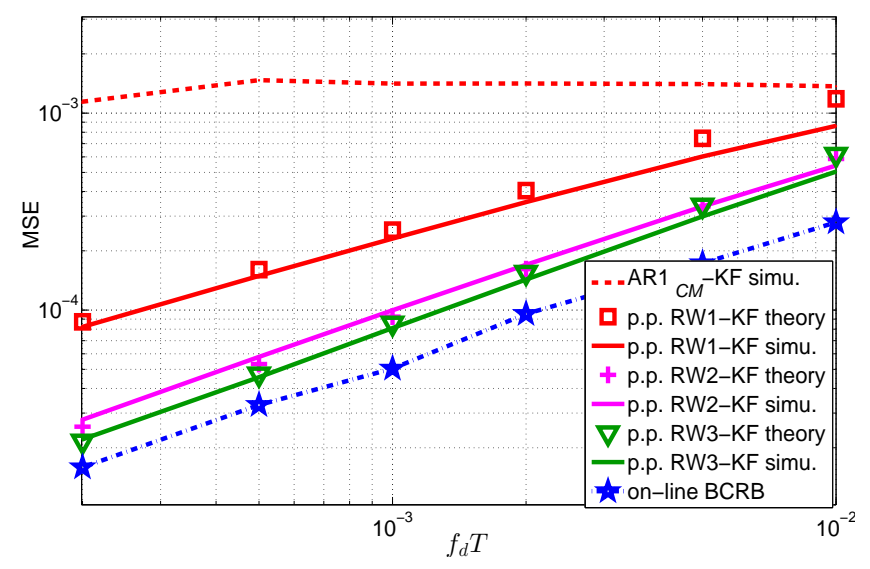

Fig. 4: MSE versus $f_{d} T$ with SNR $=20 \mathrm{~dB}, N_{p}=16$, GSM channel

performance of this low-dimensional solution is comparable to that of the joint multi-path KF. A possible way to extend this work could be by applying it to MIMO-OFDM systems.

\section{APPENDIX A}

DISCRETE-TIME EQUIVALENT CHANNEL

We assume a classical analogue "physical channel" with $L$ multi-path delays and $L$ uncorrelated multi-path CAs. The 


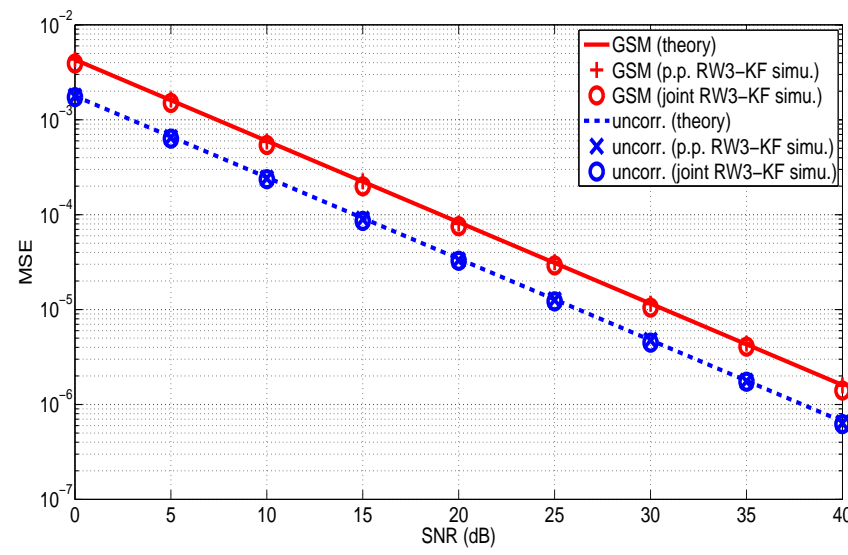

Fig. 5: MSE comparison between the per-path RW3-KF and joint multi-path RW3-KF with $f_{d} T=10^{-3}, N_{p}=16$ under different types of channels

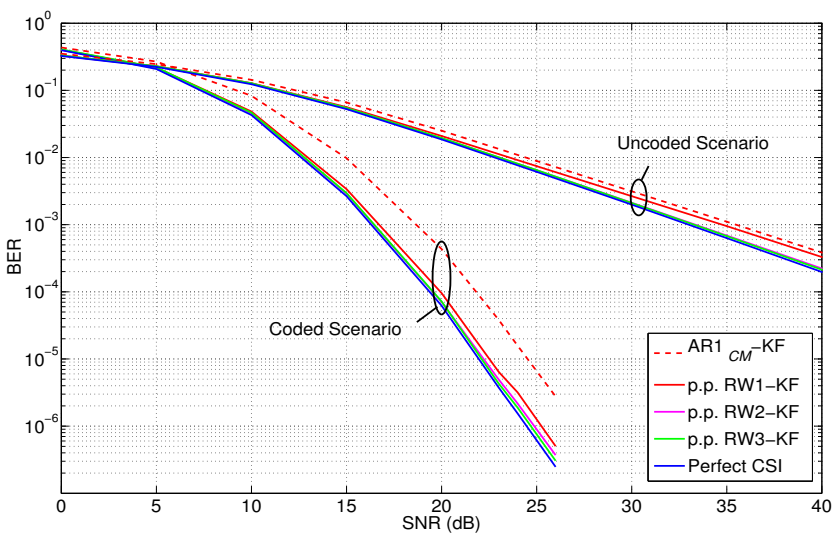

Fig. 6: BER performance for 16-QAM modulation, $f_{d} T=10^{-3}, N_{p}=8$ uncoded and coded (NRNSC) $[5,7]_{8}$ scenarios, GSM channel
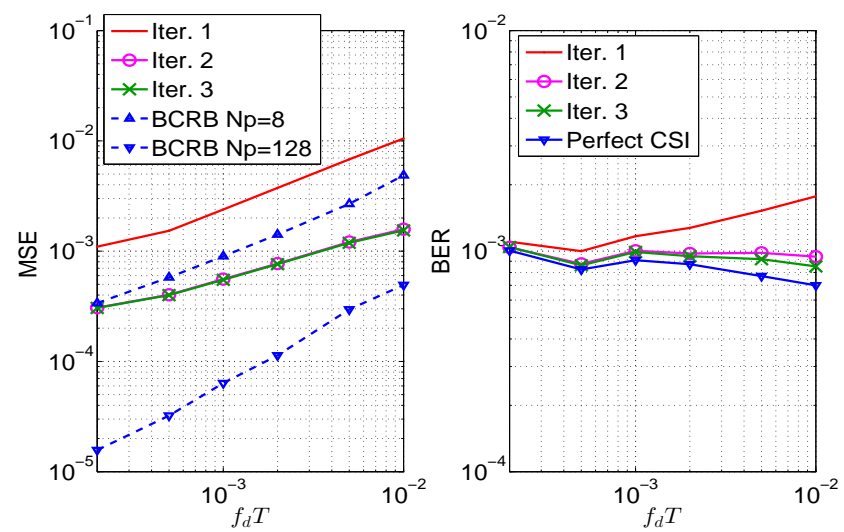

Fig. 7: MSE and BER performance (per-path RW1-KF) for 4-QAM modulation, $\mathrm{SNR}=10 \mathrm{~dB}, N_{p}=8$ coded (NRNSC) $[5,7]_{8}$ scenario with iterative detection, GSM channel

impulse response of this channel is written as [39]:

$$
h_{\text {phy }}(\tau)=\sum_{l=1}^{L} \alpha^{(l)} \delta\left(\tau-\tau^{(l)} T_{s}\right) .
$$

where delays $\tau^{(l)} T_{s}$ are not necessarily uniformly spaced every $T_{s}$. This channel can also be replaced by a DTE channel with impulse response truncated to $L^{\prime}$ coefficients $h[k]$ uniformly spaced every $T_{s}$, for $k=1,2, \cdots L^{\prime}$, obtained after limitation to a bandwidth $B=1 / T_{s}$ and sampling every $T_{s}$ :

$$
\begin{aligned}
h[k] & =h\left((k-1) T_{s}+\tau^{(1)} T_{s}\right) \\
& =\sum_{l=1}^{L} \alpha^{(l)} \operatorname{sinc}\left(\pi\left(k-1-\tau^{(l)}+\tau^{(1)}\right)\right) .
\end{aligned}
$$

with $h(\tau)=h_{\text {phy }}(\tau) * \operatorname{sinc}\left(\pi \tau / T_{s}\right), \operatorname{sinc}=\frac{\sin x}{x}$ and $*$ the convolution operator. Without loss of generality, we assume that $\tau^{(1)}=0$. This equation tells that each coefficient $h[k]$ can be linked to the $L$ physical CAs. Thus we can conclude:

1) The DTE channel is uncorrelated if and only if the physical path delays $\tau^{(l)} T_{s}$ are multiples of the sampling time $T_{s}$, i.e., if $\tau^{(l)}$ are integers. Indeed, without this condition, the $L^{\prime}$ taps $h[k]$ of the DTE channel are correlated, since each tap is a function of the $L$ CAs $\alpha^{(l)}, l=1 \ldots L$. However, in the special case where $\tau^{(l)}$ are integers, only $L$ taps among $L^{\prime}$ are non-zero. Those $L$ coefficients of $h[k]$ are uncorrelated w.r.t. one another, and coincide with the $L$ physical CAs, amounting to an uncorrelated DTE channel.

2) The condition where $\tau^{(l)}, l=1, \ldots, L$ are integers is equivalent to the condition $\mathbf{F}_{\mathbf{p}}^{H} \mathbf{F}_{\mathbf{p}}=N_{p} \mathbf{I}_{L}$ in our model assumption. Indeed, according to the comb-type pilot model with $N_{p}$ pilots spaced every $L_{f}=N / N_{p}$ subcarriers, the non-diagonal elements of the matrix (for $\left.l \neq l^{\prime}\right)$ is calculated by:

$$
\begin{aligned}
{\left[\mathbf{F}_{\mathbf{p}}^{H} \mathbf{F}_{\mathbf{p}}\right]_{l, l^{\prime}} } & =\sum_{k=0}^{N_{p}-1} e^{j 2 \pi\left(\frac{k}{N_{p}}-\frac{1}{2}\right)\left(\tau^{(l)}-\tau^{\left(l^{\prime}\right)}\right)} \\
& =e^{-j \pi \Delta \tau} \sum_{k=0}^{N_{p}-1}\left(e^{\frac{j 2 \pi \Delta \tau}{N_{p}}}\right)^{k} \\
& =\frac{2 j}{e^{\frac{j 2 \pi \Delta \tau}{N_{p}}}-1} \cdot \sin (\pi \Delta \tau) .
\end{aligned}
$$

So, the coefficients $\left[\mathbf{F}_{\mathbf{p}}^{H} \mathbf{F}_{\mathbf{p}}\right]_{l, l^{\prime}}=0$ if and only if $\Delta \tau=$ $\tau^{(l)}-\tau^{\left(l^{\prime}\right)}$ are integers. By using $\tau^{(1)}=0, \tau^{(l)}$ should be integers.

Finally, we can conclude that the DTE channel is uncorrelated if and only if the condition $\mathbf{F}_{\mathbf{p}}^{H} \mathbf{F}_{\mathbf{p}}=N_{p} \mathbf{I}_{L}$ is satisfied.

\section{APPENDIX B}

\section{TRANSFORMation OF THE KALMAN GaIN WITH THE WOODBURY IDENTITIES}

In this section, we use the following two identities [40, p. 1348] to develop the deduction:

Woodbury's identity 1:

$$
\left(\mathbf{A B} \mathbf{A}^{H}+\sigma^{2} \mathbf{I}\right)^{-1}=\frac{1}{\sigma^{2}}\left[\mathbf{I}-\mathbf{A}\left(\mathbf{A}^{H} \mathbf{A}+\sigma^{2} \mathbf{B}^{-1}\right)^{-1} \mathbf{A}^{H}\right]
$$

Woodbury's identity 2:

$$
\left(\mathbf{A}^{-1}+\mathbf{B}^{H} \mathbf{C B}\right)^{-1}=\mathbf{A}-\mathbf{A} \mathbf{B}^{H}\left(\mathbf{B} \mathbf{A} \mathbf{B}^{H}+\mathbf{C}\right)^{-1} \mathbf{B A}
$$

We rewrite the Kalman gain of Equation (16) as:

$\mathbf{K}_{(k)}=\mathbf{P}_{(k \mid k-1)} \mathbf{F}_{\mathbf{s}}^{H}\left(\mathbf{F}_{\mathbf{s}} \mathbf{P}_{(k \mid k-1)} \mathbf{F}_{\mathbf{s}}^{H}+\sigma_{\mathrm{w}}^{2} \mathbf{I}_{N_{p}}\right)^{-1}$ 


$$
\begin{aligned}
\stackrel{(50)}{=} & \mathbf{P}_{(k \mid k-1)} \mathbf{F}_{\mathbf{s}}^{H} \frac{1}{\sigma_{\mathrm{w}}^{2}}\left[\mathbf{I}_{N_{p}}-\mathbf{F}_{\mathbf{s}}\left(\mathbf{F}_{\mathbf{s}}^{H} \mathbf{F}_{\mathbf{s}}+\sigma_{\mathrm{w}}^{2} \mathbf{P}_{(k \mid k-1)}^{-1}\right)^{-1} \mathbf{F}_{\mathbf{s}}^{H}\right] \\
= & \frac{1}{\sigma_{\mathrm{w}}^{2}} \mathbf{P}_{(k \mid k-1)}\left(\mathbf{F}_{\mathbf{p}} \mathcal{S}\right)^{H}\left[\mathbf{I}_{N_{p}}-\mathbf{F}_{\mathbf{p}} \mathcal{S}\left(\left(\mathbf{F}_{\mathbf{p}} \mathcal{S}\right)^{H}\left(\mathbf{F}_{\mathbf{p}} \mathcal{S}\right)\right.\right. \\
& \left.\left.+\sigma_{\mathrm{w}}^{2} \mathbf{P}_{(k \mid k-1)}^{-1}\right)^{-1}\left(\mathbf{F}_{\mathbf{p}} \mathcal{S}\right)^{H}\right] \\
= & \frac{1}{\sigma_{\mathrm{w}}^{2}} \mathbf{P}_{(k \mid k-1)} \mathcal{S}^{H}\left[\mathbf{F}_{\mathbf{p}}^{H}-\mathbf{F}_{\mathbf{p}}^{H} \mathbf{F}_{\mathbf{p}} \mathcal{S}\left(\mathcal{S}^{H} \mathbf{F}_{\mathbf{p}}^{H} \mathbf{F}_{\mathbf{p}} \mathcal{S}\right.\right. \\
& \left.\left.+\sigma_{\mathrm{w}}^{2} \mathbf{P}_{(k \mid k-1)}^{-1}\right)^{-1} \mathcal{S}^{H} \mathbf{F}_{\mathbf{p}}^{H}\right] \\
= & \frac{1}{\sigma_{\mathrm{w}}^{2}} \mathbf{P}_{(k \mid k-1)} \mathcal{S}^{H}\left[\mathbf{I}_{L}-\mathbf{F}_{\mathbf{p}}^{H} \mathbf{F}_{\mathbf{p}} \mathcal{S}\left(\mathcal{S}^{H} \mathbf{F}_{\mathbf{p}}^{H} \mathbf{F}_{\mathbf{p}} \mathcal{S}\right.\right. \\
& \left.\left.+\sigma_{\mathrm{w}}^{2} \mathbf{P}_{(k \mid k-1)}^{-1}\right)^{-1} \mathcal{S}^{H}\right] \mathbf{F}_{\mathbf{p}}^{H} .
\end{aligned}
$$

Let $\mathbf{A}=\mathbf{F}_{\mathbf{p}}^{H} \mathbf{F}_{\mathbf{p}}, \mathbf{B}=\mathcal{S}^{H}, \mathbf{C}=\sigma_{\mathrm{w}}^{2} \mathbf{P}_{(k \mid k-1)}^{-1}$, and we continue the deduction:

$$
\begin{aligned}
\mathbf{K}_{(k)}= & \frac{1}{\sigma_{\mathrm{w}}^{2}} \mathbf{P}_{(k \mid k-1)} \mathcal{S}^{H}\left[\mathbf{I}_{L}-\mathbf{A} \mathbf{B}^{H}\left(\mathbf{B} \mathbf{A} \mathbf{B}^{H}+\mathbf{C}\right)^{-1} \mathbf{B}\right] \mathbf{F}_{\mathbf{p}}^{H} \\
= & \frac{1}{\sigma_{\mathrm{w}}^{2}} \mathbf{P}_{(k \mid k-1)} \mathcal{S}^{H}\left[\mathbf{A}-\mathbf{A} \mathbf{B}^{H}\left(\mathbf{B} \mathbf{A} \mathbf{B}^{H}+\mathbf{C}\right)^{-1} \mathbf{B} \mathbf{A}\right] \mathbf{A}^{-1} \mathbf{F}_{\mathbf{p}}^{H} \\
\stackrel{(51}{=} & \frac{1}{\sigma_{\mathrm{w}}^{2}} \mathbf{P}_{(k \mid k-1)} \mathcal{S}^{H}\left(\mathbf{A}^{-1}+\mathbf{B}^{H} \mathbf{C}^{-1} \mathbf{B}\right)^{-1} \mathbf{A}^{-1} \mathbf{F}_{\mathbf{p}}^{H} \\
= & \frac{1}{\sigma_{\mathbf{w}}^{2}} \mathbf{P}_{(k \mid k-1)} \mathcal{S}^{H}\left(\left(\mathbf{F}_{\mathbf{p}}^{H} \mathbf{F}_{\mathbf{p}}\right)^{-1}+\frac{1}{\sigma_{\mathrm{w}}^{2}} \mathcal{S} \mathbf{P}_{(k \mid k-1)} \mathcal{S}^{H}\right)^{-1} \\
& \left(\mathbf{F}_{\mathbf{p}}^{H} \mathbf{F}_{\mathbf{p}}\right)^{-1} \mathbf{F}_{\mathbf{p}}^{H} \\
= & \mathbf{P}_{(k \mid k-1)} \mathcal{S}^{H}\left(\left(\mathbf{F}_{\mathbf{p}}^{H} \mathbf{F}_{\mathbf{p}}\right)^{-1} \sigma_{\mathrm{w}}^{2}+\mathcal{S} \mathbf{P}_{(k \mid k-1)} \mathcal{S}^{H}\right)^{-1} \\
& \left(\mathbf{F}_{\mathbf{p}}^{H} \mathbf{F}_{\mathbf{p}}\right)^{-1} \mathbf{F}_{\mathbf{p}}^{H} \\
= & \mathbf{K}_{\mathbf{e q}(k)} \cdot\left(\mathbf{F}_{\mathbf{p}}^{H} \mathbf{F}_{\mathbf{p}}\right)^{-1} \mathbf{F}_{\mathbf{p}}^{H}
\end{aligned}
$$

with $\mathbf{K}_{\mathbf{e q}(k)}$ defined by:

$$
\mathbf{K}_{\mathbf{e q}(k)}=\mathbf{P}_{(k \mid k-1)} \mathcal{S}^{H}\left(\left(\mathbf{F}_{\mathbf{p}}^{H} \mathbf{F}_{\mathbf{p}}\right)^{-1} \sigma_{\mathrm{w}}^{2}+\mathcal{S} \mathbf{P}_{(k \mid k-1)} \mathcal{S}^{H}\right)^{-1}
$$

\section{ACKNOWLEDGMENT}

The authors would like to thank the associate editor and anonymous reviewers for their helpful comments and suggestions of this manuscript.

This work has been performed with the support of the ANR-TDM national project CORRIDOR (COgnitive Radio for RaIlway through Dynamic and Opportunistic spectrum Reuse) and the Regional project CISIT (Campus International pour la securite et lintermodalite dans les transports) financed by the North region and the European program FEDER.

\section{REFERENCES}

[1] Y. Zhao and A. Huang, "A novel channel estimation method for OFDM mobile communication systems based on pilot signals and transformdomain processing," in IEEE 47th Vehicular Technology Conference, 1997, vol. 3, 1997, pp. 2089-2093 vol.3.

[2] M.-H. Hsieh and C.-H. Wei, "Channel estimation for OFDM systems based on comb-type pilot arrangement in frequency selective fading channels," IEEE Trans. Consum. Electron., vol. 44, no. 1, pp. 217-225, 1998.
[3] S. Coleri, M. Ergen, A. Puri, and A. Bahai, "Channel estimation techniques based on pilot arrangement in OFDM systems," IEEE Trans. Broadcast., vol. 48, no. 3, pp. 223-229, 2002.

[4] C. Komninakis, C. Fragouli, A. Sayed, and R. Wesel, "Multi-input multi-output fading channel tracking and equalization using Kalman estimation," IEEE Trans. Signal Process., vol. 50, no. 5, pp. 1065-1076, May 2002.

[5] W. Chen and R. Zhang, "Kalman-filter channel estimator for OFDM systems in time and frequency-selective fading environment," in IEEE International Conference on Acoustics, Speech, and Signal Processing, 2004. (ICASSP '04)., vol. 4, May 2004, pp. iv-377-iv-380 vol.4.

[6] T. Al-Naffouri, "An EM-Based Forward-Backward Kalman Filter for the Estimation of Time-Variant Channels in OFDM," IEEE Trans. Signal Process., vol. 55, no. 7, pp. 3924-3930, 2007.

[7] H. Hijazi and L. Ros, "Joint data QR-detection and Kalman estimation for OFDM time-varying Rayleigh channel complex gains," IEEE Trans. Commun., vol. 58, no. 1, pp. 170-178, Jan. 2010.

[8] E. P. Simon, L. Ros, H. Hijazi, and M. Ghogho, "Joint Carrier Frequency Offset and Channel Estimation for OFDM Systems via the EM Algorithm in the Presence of Very High Mobility," IEEE Trans. Signal Process., vol. 60, no. 2, pp. 754-765, Feb. 2012.

[9] S. Hwang, J. Um, M. Song, C. Kim, H. Park, and Y. Kim, "Design and verification of IEEE 802.22 WRAN physical layer," in 3rd International Conference on Cognitive Radio Oriented Wireless Networks and Communications, 2008.

[10] M. Sohail and T. Al-Naffouri, "An EM based frequency domain channel estimation algorithm for multi-access OFDM systems," ELSEVIER Signal Processing, vol. 90, no. 5, pp. 1562 - 1572, 2010.

[11] B. Yang, K. Letaief, R. Cheng, and C. Z., "Channel estimation for OFDM transmission in multipath fading channels based on parametric channel modeling," IEEE Trans. Commun., vol. 49, no. 3, pp. 467-479, 2001.

[12] M. R. Raghavendra, S. Bhashyam, and K. Giridhar, "Exploiting hopping pilots for parametric channel estimation in OFDM systems," IEEE Signal Processing Letters, vol. 12, no. 11, pp. 737-740, 2005.

[13] A. Barbieri, A. Piemontese, and G. Colavolpe, "On the ARMA approximation for fading channels described by the Clarke model with applications to Kalman-based receivers," IEEE Trans. Wireless Commun., vol. 8, no. 2, pp. 535-540, 2009.

[14] S. Ghandour-Haidar, L. Ros, and J.-M. Brossier, "On the use of firstorder autoregressive modeling for Rayleigh flat fading channel estimation with Kalman filter," Signal Processing, vol. 92, no. 2, pp. 601 606, 2012.

[15] L. Ros, S. Ghandour-Haidar, and J.-M. Brossier, "Sur l'utilisation des filtres du premier-ordre pour l'estimation d'un canal radio-mobile de Rayleigh à évanouissement plat," in XXIIIème colloque GRETSI (GRETSI 2011), Bordeaux : France, 2011.

[16] R. Gerzaguet, L. Ros, J.-M. Brossier, S. Ghandour-Haidar, and F. Belvèze, "Self-adaptive stochastic rayleigh flat fading channel estimation," in IEEE International Conference on Digital Signal Processing (DSP), 2013.

[17] L. Ros and E. P. Simon, "Second-order modeling for Rayleigh flat fading channel estimation with Kalman Filter," in 17th International Conference on Digital Signal Processing (DSP), 2011, pp. 1-6.

[18] H. Shu, E. P. Simon, and L. Ros, "Third-order kalman filter: Tuning and steady-state performance," IEEE Signal Processing Letters, vol. 20, no. 11, pp. 1082-1085, 2013.

[19] J. Cai, X. Shen, and J. W. Mark, "Robust channel estimation for OFDM wireless communication systems - an $H_{\infty}$ approach," IEEE Trans. Wireless Commun., vol. 3, no. 6, pp. 2060-2071, Nov 2004.

[20] M. Karthik and D. Sreedhar, "VSSO Kalman-usage of Barhumi's pilot pattern for channel estimation in MIMO-OFDM systems," Electronics Letters, vol. 48, no. 14, pp. 883-884, July 2012.

[21] K. Muralidhar and K. H. Li, "A Low-Complexity Kalman Approach for Channel Estimation in Doubly-Selective OFDM Systems," IEEE Signal Process. Letters, vol. 16, no. 7, pp. 632-635, July 2009.

[22] K. Muralidhar, "Equivalence of VSSO and VSVO Kalman Channel Estimators in Doubly-Selective OFDM Systems - A Theoretical Perspective," IEEE Signal Process. Letters, vol. 18, no. 4, pp. 223-226, April 2011.

[23] K. Muralidhar and D. Sreedhar, "Pilot Design for Vector State-Scalar Observation Kalman Channel Estimators in Doubly-Selective MIMOOFDM Systems," IEEE Wireless Commun. Letters, vol. 2, no. 2, pp. 147-150, April 2013.

[24] P. Banelli, R. Cannizzaro, and L. Rugini, "Data-Aided Kalman Tracking for Channel Estimation in Doppler-Affected OFDM Systems," in IEEE 
International Conference on Acoustics, Speech and Signal Processing, vol. 3, April 2007, pp. III-133-III-136.

[25] R. Cannizzaro, P. Banelli, and G. Leus, "Adaptive Channel Estimation for OFDM Systems with Doppler spread," in IEEE 7th Workshop on Signal Processing Advances in Wireless Communications, July 2006, pp. $1-5$.

[26] N. Chen, J. Zhang, and P. Zhang, "Improved Channel Estimation Based on Parametric Channel Approximation Modeling for OFDM Systems," IEEE Trans. Broadcast., vol. 54, no. 2, pp. 217-225, June 2008.

[27] H. Hijazi and L. Ros, "Polynomial Estimation of Time-Varying Multipath Gains With Intercarrier Interference Mitigation in OFDM Systems," IEEE Trans. Veh. Technol., vol. 58, no. 1, pp. 140-151, 2009.

[28] L. Ros, H. Hijazi, and E. P. Simon, "Complex amplitudes tracking loop for multipath channel estimation in OFDM systems under slow to moderate fading," ELSEVIER Signal Processing, vol. 97, pp. 134-145, 2014.

[29] W. Jakes, Microwave Mobile Communications. New-York: John Wiley, 1974.

[30] H. Hijazi and L. Ros, "Bayesian Cramera-Rao Bounds for complex gain parameters estimation of slowly varying Rayleigh channel in OFDM systems," ELSEVIER Signal Processing, vol. 89, no. 1, pp. 111 - 115, 2009.

[31] S. M. Kay, Fundamentals of Statistical Signal Processing : Estimation Theory. Prentice Hall PTR, April 5, 1993.

[32] E. Kaplan and C. Hegarty, Understanding GPS: Principles and Applications, Second Edition. Artech House Publishers, 2005.

[33] L. Ros, H. Hijazi, and E. P. Simon, "Paths complex gain tracking algorithms for ofdm receiver in slowly-varying channels," in Communications, Control and Signal Processing (ISCCSP), 2010 4th International Symposium on, Mar. 2010, pp. 1-6.

[34] I. Barhumi, G. Leus, and M. Moonen, "Optimal training design for MIMO OFDM systems in mobile wireless channels," IEEE Trans. Signal Process., vol. 51, no. 6, pp. 1615-1624, June 2003.

[35] J. G. Andrews, A. Ghosh, and R. Muhamed, Fundamentals of WiMAX: Understanding Broadband Wireless Networking. Prentice Hall PTR, 2007.

[36] European Telecommunications Standards Institute, European Digital Cellular Telecommunication System (Phase 2), Radio Transmission and Reception, GSM 05.05, vers. 4.6.0.

[37] E. Simon and M. Khalighi, "Iterative Soft-Kalman Channel Estimation for Fast Time-Varying MIMO-OFDM Channels," IEEE Wireless Commun. Letters, vol. 2, no. 6, pp. 599-602, December 2013.

[38] E. Simon, L. Ros, H. Hijazi, J. Fang, D. Gaillot, and M. Berbineau, "Joint Carrier Frequency Offset and Fast Time-Varying Channel Estimation for MIMO-OFDM Systems," IEEE Trans. Veh. Technol., vol. 60, no. 3, pp. 955-965, 2011.

[39] J. Proakis, Digital Communications. McGraw-Hill, third edition, 1995.

[40] H.-L. Van Trees, Optimum Array Processing: Part IV of Detection, Estimation, and Modulation Theory. John Wiley \& Sons, Inc., 2002.

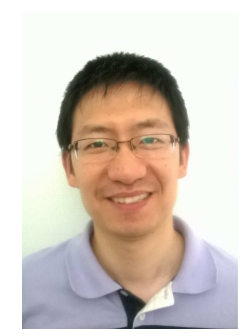

carrier systems.
Huaqiang Shu received the B.S. degree in information engineering from Tongji University, Shanghai, China in 2005, and the M.S. and Ph.D. degrees in telecommunications from Université Lille I, Science et Technologie, Villeneuve d'Ascq, France, in 2010 and 2013. He is currently a postdoctoral fellow in Gipsa-lab, Department of Image and Signal (DIS), Saint Martin d'Hères, France .

His main research interests are channel estimation algorithms for wireless communications systems and Faster-than-Nyquist (FTN) transmission in Multi-

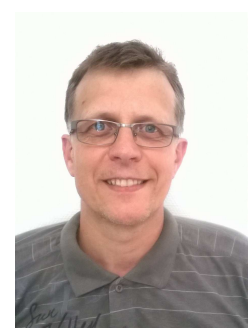

Laurent Ros received the degree in electrical engineering from the Ecole Supérieure d'Electricité (Supélec), Paris, France, in 1992 and the Ph.D. degree in signal processing and communications from Grenoble Institute of Technology (GrenobleINP), Grenoble, France, in 2001. From 1993 to 1995, he was with France-Telecom R\&D center, Lannion, France, where he worked in the area of very low frequency transmissions for submarine applications, in collaboration with Direction of Naval Construction, Toulon, France. From 1995 to 1999 he was a Research and Development team manager at Sodielec, Millau, France, where he worked in the design of digital modems and audio codecs for telecommunication applications. Since 1999, he has joined the Gipsalaboratory/DIS (ex "Laboratory of Image and Signal") and Grenoble-INP where he is currently an Associate Professor.

His research interests are in statistical signal processing for communication, including channel estimation and synchronization problems for wireless communications.

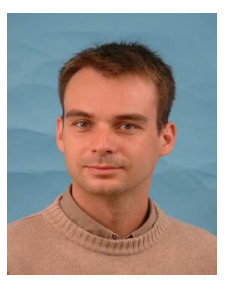

Eric P. Simon received the Master's degree in electronics engineering from the Superior School of Electronics (ESCPE), Lyon, France, in 1999, and the Ph.D. degree in signal processing and communications from the National Polytechnic Institute of Grenoble (INPG), France, in 2004. During 2005, he was a Teaching Assistant at the INPG and the following year he joined one of France Telecom R\&D Laboratories as a Postdoctoral Fellow.

$\mathrm{He}$ is currently an Associate Professor at the Institute of Electronics, Microelectronics and Nanotechnology (IEMN), TELICE (Telecommunications, Interference and Electromagnetic Compatibility) Group, University of Lille, France. His main research interests are in mobile communications and carrier and symbol synchronization. 